Florida International University

FIU Digital Commons

6-26-2020

\title{
Online Coordination Mechanism for Road Infrastructure Restoration using Unmanned Aerial Vehicles
}

Mohammad Amin Farzaneh

mfarz004@fiu.edu

Follow this and additional works at: https://digitalcommons.fiu.edu/etd

Part of the Operations Research, Systems Engineering and Industrial Engineering Commons

\section{Recommended Citation}

Farzaneh, Mohammad Amin, "Online Coordination Mechanism for Road Infrastructure Restoration using Unmanned Aerial Vehicles" (2020). FIU Electronic Theses and Dissertations. 4507.

https://digitalcommons.fiu.edu/etd/4507

This work is brought to you for free and open access by the University Graduate School at FIU Digital Commons. It has been accepted for inclusion in FIU Electronic Theses and Dissertations by an authorized administrator of FIU Digital Commons. For more information, please contact dcc@fiu.edu. 


\title{
FLORIDA INTERNATIONAL UNIVERSITY
}

Miami, Florida

\section{ONLINE COORDINATION MECHANISM FOR ROAD INFRASTRUCTURE RESTORATION USING UNMANNED AERIAL VEHICLES}

\author{
A thesis submitted in partial fulfillment of \\ the requirements for the degree of \\ MASTER OF SCIENCE \\ in \\ ENGINEERING MANAGEMENT \\ by
}

Mohammad Amin Farzaneh 
To: Dean John L. Volakis

College of Engineering and Computing

This thesis, written by Mohammad Amin Farzaneh, and entitled Online Coordination Mechanism for Road Infrastructure Restoration using Unmanned Aerial Vehicles, having been approved in respect to style and intellectual content, is referred to you for judgment.

We have read this thesis and recommend that it be approved.

Shih-ming (Ted) Lee

Mohammadhadi Amini

Shabnam Rezapour, Major Professor

Date of Defense: June 26, 2020

The thesis of Mohammad Amin Farzaneh is approved.

Dean John L. Volakis College of Engineering and Computing

Andrés G. Gil

Vice President for Research and Economic Development and Dean of the University Graduate School

Florida International University, 2020 
(C) Copyright 2020 by Mohammad Amin Farzaneh

All rights reserved. 


\section{DEDICATION}

This thesis is dedicated to my parents for their endless love, support, and encouragement throughout my life. 


\section{ACKNOWLEDGMENTS}

I would like to sincerely thank my committee for their support and patience. I have to thank Dr. Rezapour, in a special way, I express my heartfelt gratefulness for her guide throughout this study. My younger brothers and friends deserve my wholehearted thanks as well. 


\section{ABSTRACT OF THE THESIS \\ ONLINE COORDINATION MECHANISM FOR ROAD INFRASTRUCTURE \\ RESTORATION USING UNMANNED AERIAL VEHICLES}

by

Mohammad Amin Farzaneh

Florida International University, 2020

Miami, Florida

Professor Shabnam Rezapour, Major Professor

The goal of this thesis is to study two barriers of efficient road network restoration, namely, the lack of debris information and the lack of coordination among the restoration operations. We develop an integrative online optimization model with a model-based data diffusion component to coordinate three restoration-interdependent operations in the disaster response phases such as damage assessment, road recovery, and relief distribution. The model developed for the damage assessment operation controls the debris data diffusion speed in the integrative framework. This data is periodically shared with an online model developed to prioritize the recovery process for blocked roads. Road prioritization is done in a way to make the highest acceleration in the relief distribution operation. The integrative framework is tested on the road network of Miami-Dade and its performance is compared with an online heuristic benchmark mimicking the performance of the Federal Emergency Management Agency. 


\section{TABLE OF CONTENTS}

CHAPTER

PAGE

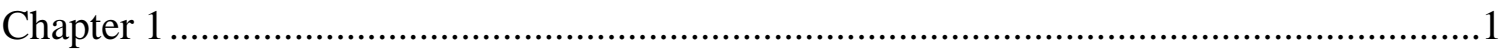

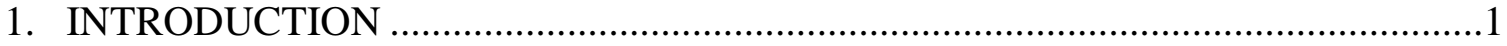

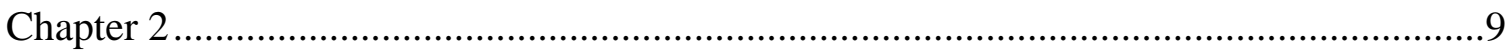

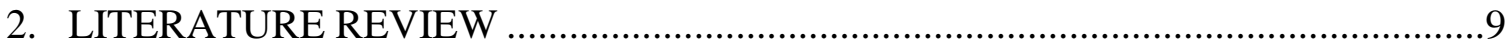

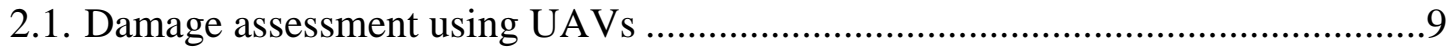

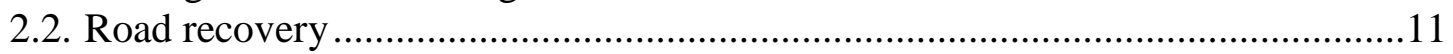

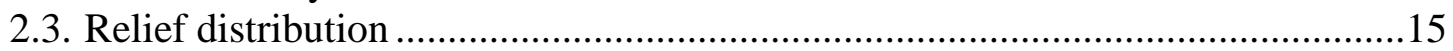

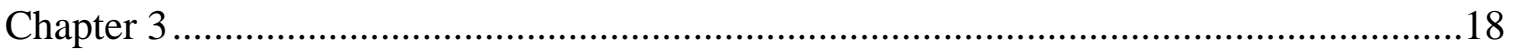

3. MODEL FORMULATION AND SOLUTION APPROACH .................................18

3.1. Model for prepositioning damage assessment facilities ..................................19

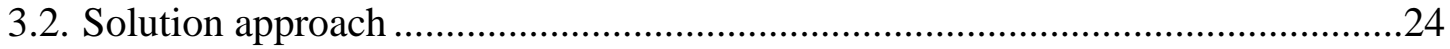

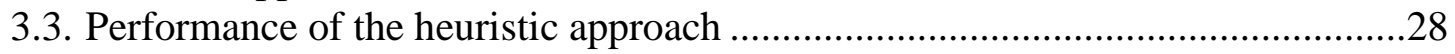

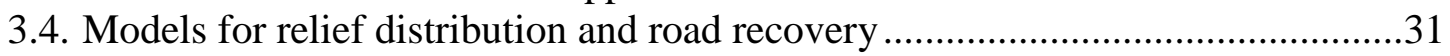

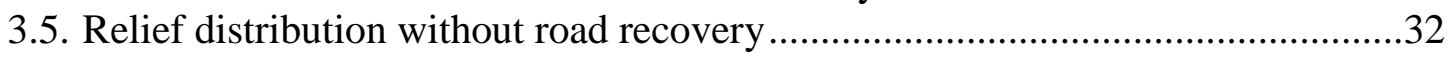

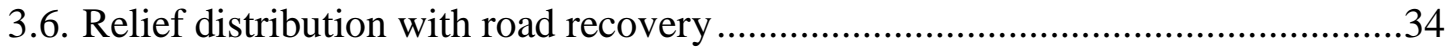

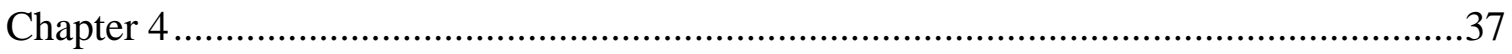

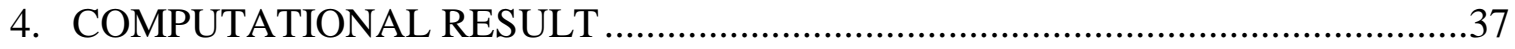

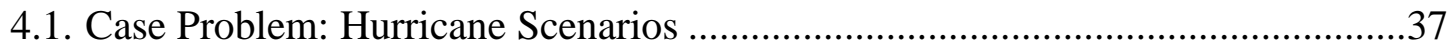

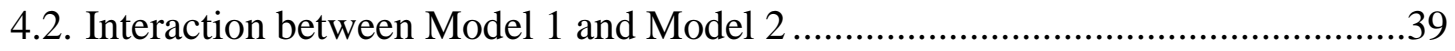

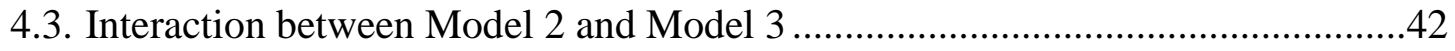

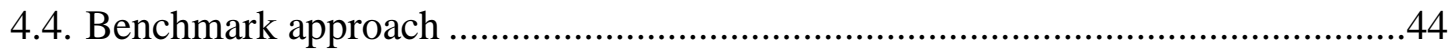

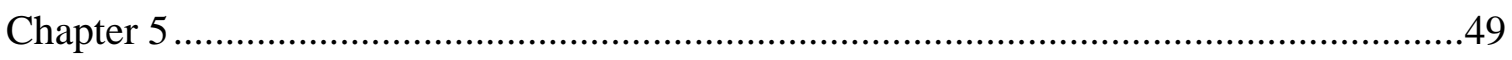

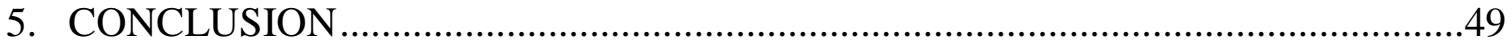

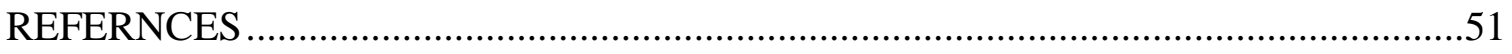

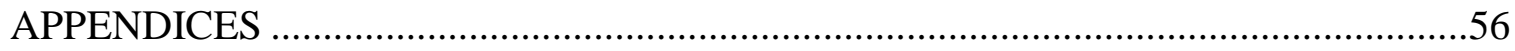




\section{LIST OF TABLES}

TABLE

PAGE

Table 1. The accuracy of the solutions provided by the heuristic approach .....................30

Table 2. The results for the problem instances ......................................................40

Table 3. The results for integrative and non-integrative approaches ............................44

Table 4. The results for the integrative and benchmark approaches.............................46

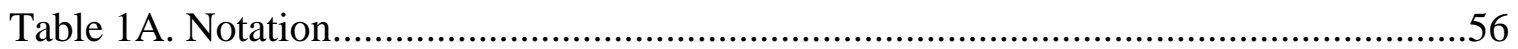




\section{TABLE OF FIGURES}

FIGURE

PAGE

Figure 1. Disaster recovery timeline (FEMA 2018) ...............................................

Figure 2. The interdependencies between the models ...............................................5

Figure 3. The structure of the integrative framework ..............................................

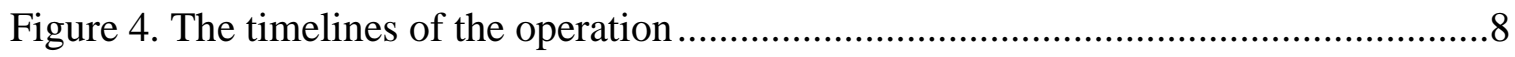

Figure 5. Network reformation for a sample road network ......................................21

Figure 6. The super-network constructed for a sample unified network ........................26

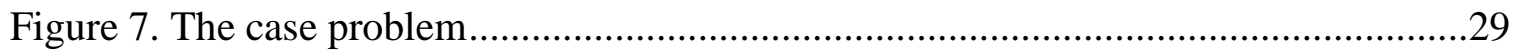

Figure 8. The tradeoff between $\Psi$ and the pre-disaster cost in the case network .............31

Figure 9. The zones of disrupted nodes and roads in the case problem..........................38

Figure 10. Variations in the total response cost ..................................................41

Figure 11. Increment rates of costs with respect to the scan completion time .................42

Figure 12. The improvement in the response cost …...........................................43

Figure 13. The differences between the post-disaster response costs...........................45

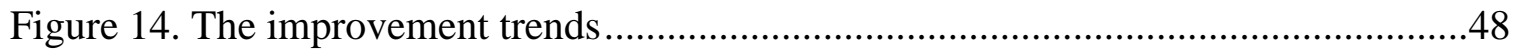

Figure 15. The conditions under which the integrative approach is more effective..........50

Figure 1C. The structure of the sample network after the second reformation ...............61 


\section{Chapter 1}

\section{INTRODUCTION}

One of the most important factors that impede response operations in disasters is a disruption in road infrastructure. Blocked and damaged roads reduce accessibility to critical facilities such as stocks of emergency supplies, hospitals, and shelters. They slow down relief transportation (of emergency goods, casualties, rescue teams, etc.) between a set of supply nodes (such as stocks of prepositioned goods and airports) and demand nodes (such as affected sites and hospitals). In the 2015 Nepal Earthquake, some roads close to the epicenter were severely damaged and several villages became inaccessible for days. Similarly, in the 2011 Japan tsunami, debris accumulation blocked several roads in the downtown of Kamaishi City and deterred rescue operations for the affected population (Kasaei \& Salman 2016). In disaster-prone countries such as Japan, contracts with construction companies are made to dispatch recovery teams (equipped with heavy machinery such as bulldozers, draining pump vehicles and skilled personnel) within a short time after disasters to restore primary road infrastructure and accelerate response operations (Akbari \& Salman 2017).

In the US, FEMA released the National Disaster Recovery Framework (NDRF) in 2011 to better coordinate the flow of resources (money, material, and manpower) after disasters (FEMA 2018). NDRF establishes six different Recovery Support Functions (RSFs): community planning and capacity building; economic recovery; health and social services; housing; infrastructure systems; and natural and cultural resources. The mission 
of the health and social services RSF is to support locally-led recovery efforts to address public needs and health according to the timeline shown in Figure 1.

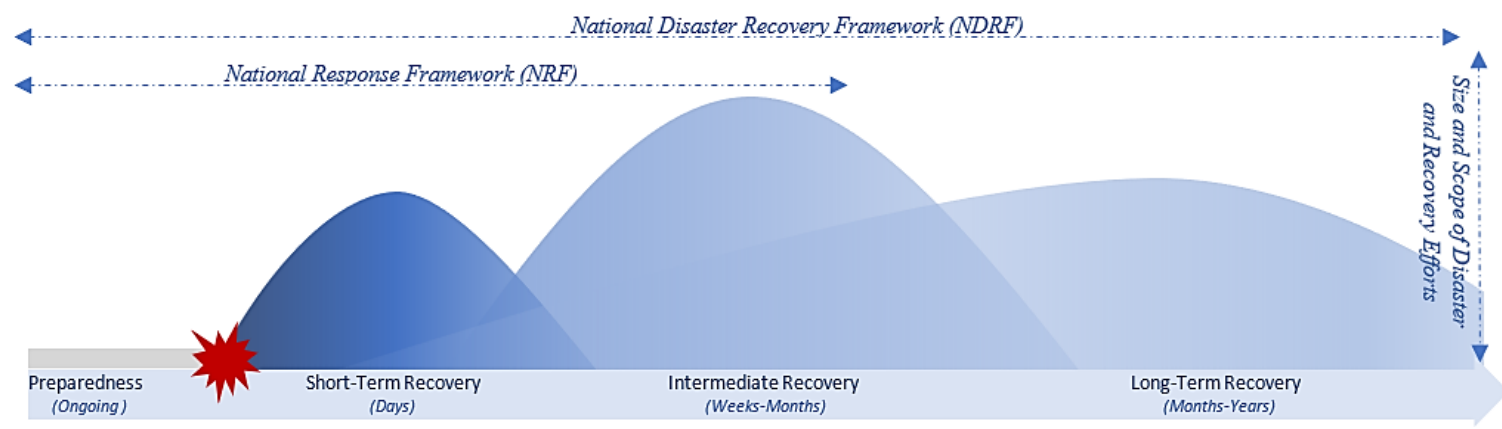

Figure 1. Disaster recovery timeline (FEMA 2018).

Clearing/repairing primary transportation roads, the problem investigated in this study, and providing shelter to displaced households are categorized as short-term recovery. Intermediate recovery includes providing accessible housing solutions and developing a comprehensive restoration plan for the affected area. The most important examples of long-term recovery are developing permanent housing and facilitating funding for business rebuilding. In this study, the focus is on the short-term recovery of the primary transportation roads. After disasters, to accelerate the relief transportation in the response phase, fast and efficient recovery of primary roads is crucial. Scarcity of recovery teams complicates the clearing/repairing process that includes several decisions (Oruc \& Kara 2018):

1) Damage Assessment: Damage assessment is necessary to accurately estimate the damage/debris levels and required recovery times for blocked roads. The damage information can be obtained using satellite images or pictures taken from the affected area using helicopters and unmanned aerial vehicles (UAVs) (Akbari \& Salman 2017; Coleman et al. 2009). Helicopter and satellite inspection at the stand-off range is fast 
but does not produce high-quality information. In contrast, UAVs are piloted remotely and can get closer to damaged infrastructure for precise scanning. High-resolution images of UAVs offer a complete aerial view gathered at a safe distance. These images can be processed to estimate the required resource and time for the recovery of damaged roads. Besides, using UAVs for damage assessment is more cost-effective. One of the important applications of UAVs is the damage assessment of agricultural areas. According to the report of the Food and Agriculture Organization of the United Nations (FAO 2017), the application of UAV technology has several advantages compared to satellite imagery: (i) It can minimize errors arising from water vapor and aerosols (atmospheric effects); and (ii) It can be deployed even under the cloud cover. That is why UAV inspection is becoming a common method for damage assessment (Lim et al. 2018). UAVs gradually scan road networks in a close distance and transit pictures to a ground control center to analyze and determine the damage levels. This information is sent to recovery teams for network restoration. The number, locations, and scan routes of the UAVs are critical decisions that determine the damage assessment speed and control the data diffusion speed in the restoration process.

2) Road Selection: After receiving damage/debris data, the most appropriate subset of blocked and scanned roads should be selected for recovery (Celik et al. 2016). These roads can only be selected from the set of roads that are already scanned by the UAVs and their damage/debris levels are known (this set is updated over the time). The selection priority of the blocked roads is determined by the reductions that their recovery can make in the transportation times between supply and demand nodes. 
Recovery teams are dispatched to the selected roads to clear/repair the roads and revive them in the road network.

3) Team Allocation: team allocation deals with assigning recovery teams to the selected roads. The number of teams assigned to each road determines its recovery time. Assigning more recovery teams to a road reduces its recovery time proportionally.

The focus of this study is on the optimization of these three decisions in the restoration process of the primary transportation roads. For this purpose, three models are developed (Figure 2):

- Model 1 - prepositioning UAVs: This model prepositions damage assessment facilities, UAVs, and their charging stations in the road network of the area that is predicted to be affected by a disaster (in the pre-disaster preparedness phase). The model is a multiobjective and multi-period mixed-integer programming that minimizes the total preparedness cost of employing UAVs and establishing the charging stations. These facilities will be used after the disaster to scan the network and assess the damage/debris levels of the blocked roads. The prepositioning decisions made by Model 1 determine when each road segment will be scanned and its damage/debris information (if there is any) will be available for recovery teams.

- Model 2 - road recovery: This model uses the damage/debris information periodically provided by Model 1 to make the best recovery decisions (road selection and team allocation decisions) for the road network (directional interdependency between Model 1 and Model 2). The decisions of this model are online and updated based on the new data periodically provided by Model 1 . When the recovery process for a selected subset 
of blocked roads ends, the set of the scanned roads with known damage/debris levels is updated and new recovery decisions are made. The recovery decisions in Model 2 are made in a way to maximize the demand fulfillment speed in the relief distribution (in the forthcoming Model 3).

- Model 3 - relief transportation: This online and multi-period model uses the recovery information (set of recovered roads) provided periodically by Model 2 to make the best transportation decisions for relief needs. This model tries to fulfill relief demands in the fastest way. The set of available roads for transportation is updated periodically according to the decisions made by Model 2. There is a mutual interdependency between Model 2 and Model 3. The selection criterion used in Model 2 depends on the demand fulfillment acceleration that can be made in Model 3. Also, the acceleration that can be made in Model 3 is affected by the recovery decisions made by Model 2.

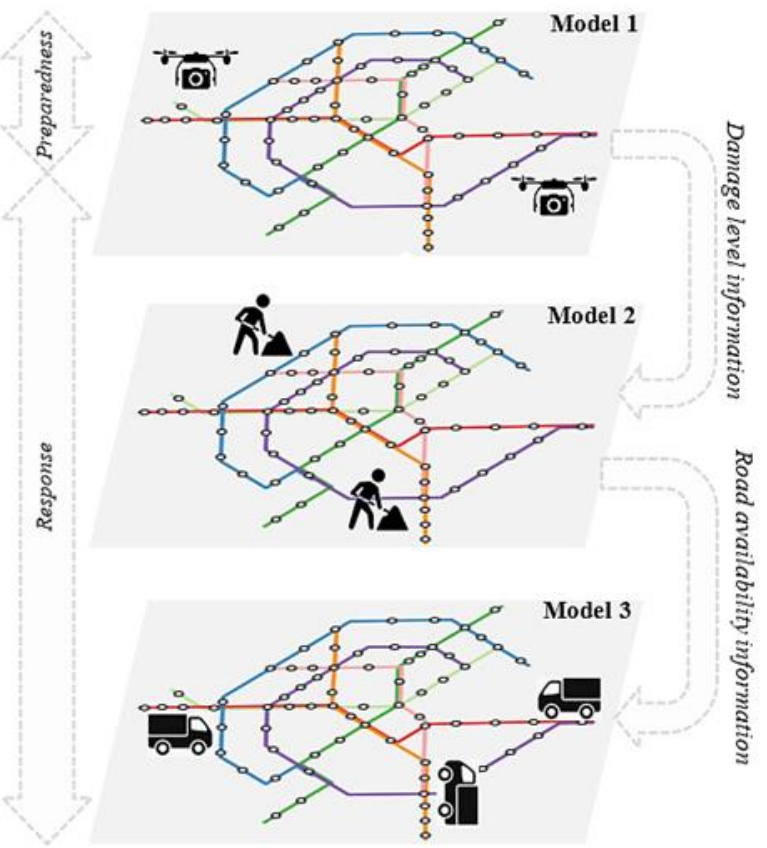

Figure 2. The interdependencies between the models. 
The lack of coordination among response operations is stated by many administrators. Altntaş and Delooz (2004) state “... coordination between operations was a major concern ..." in the aftermath of severe disasters such as the Marmara earthquake in Turkey. The information flow between these models is represented in Figure 2. The modeling framework used in this study for network restoration is online optimization with model-based data diffusion. Having a model-based data diffusion component is a new paradigm in online optimization. Online optimization is a type of optimization under incomplete information or uncertainty (Dunke \& Nickel 2016; Ghiani et al. 2004). In contrast to the stochastic or robust optimizations that rely on historical data to quantify distribution functions or variation ranges for uncertain parts (Bertsimas et al. 2011), online optimization is more compatible with chaotic circumstances after disasters that do not include any explicitly related historical records. Online optimization is focused on the realizations of input elements for which no stochastic principles are known to hold. Our framework will be a time-stamp (not sequential) online optimization in which multi-size batches of input elements (damage/debris levels) with assigned acquisition times are provided by Model 1 and automatically form a set of unprocessed input elements for Model 2. We call this modeling framework "Online Optimization with Model-based Data Diffusion $(O O M D D)$ ". Figure 3 shows the structure of this framework. The execution is carried out cyclically according to the sets of input elements. 


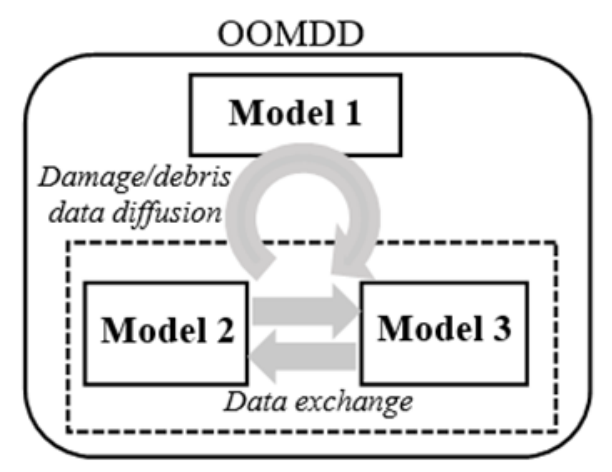

Figure 3. The structure of the integrative framework.

These three operations (damage assessment, road recovery, and relief distribution) are handled by separate organizations and there is not a single decision-maker for them. Damage assessment is performed by the Damage Assessment Team (DAT) working under the Homeland Security Department. Municipalities are usually in charge of immediate clearing/repairing of roads in urban areas. Relief distribution operation is performed by humanitarian agencies such as FEMA in the U.S. Decentralization of the operations strengthens the importance of coordination and online data exchange between the operations. Also, the timeline of these three operations is different. For example, damage assessment by UAVs is done faster than road recovery. The daily relief distribution starts immediately after the disaster and does not wait for road recovery that may last longer. Figure 4 shows that the first (damage assessment) and third (relief distribution) operations are started immediately after the disaster. The second operation (road recovery) starts when the first set of damage information is provided by the first operation. After recovering the first subset of damaged roads, the new road recovery decisions are made based on new damage information that is provided by the first operation. At the same time, the 
transportation paths of the third operations are updated according to the availability of the recently recovered roads.

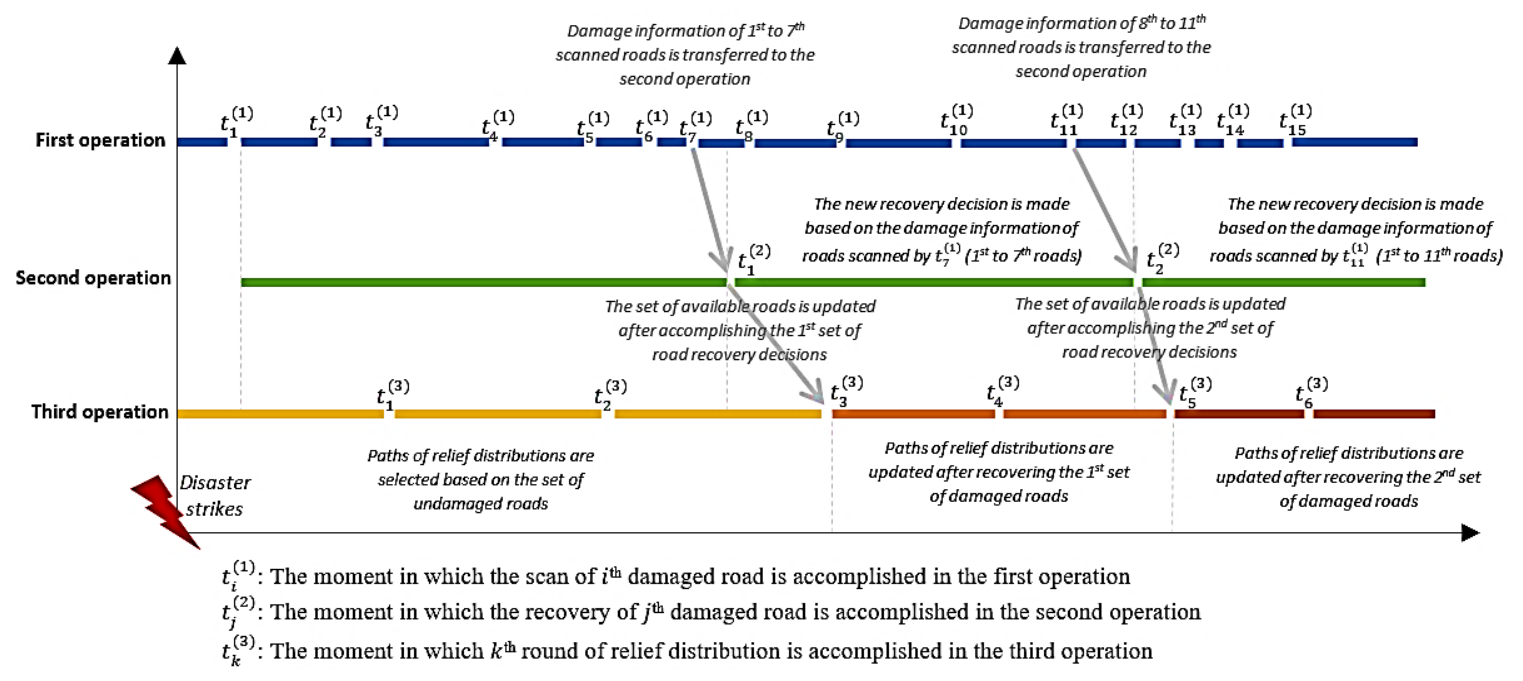

Figure 4. The timelines of the operations. 


\section{Chapter 2}

\section{LITERATURE REVIEW}

Humanitarian logistics have rich literature in operations management and management science. Recent reviews, conducted by Gupta et al. (2016), Apte (2009) and Altay and Green (2006), classify the clean/repair of primary roads as one of the major response/recovery operations and highlight it as an important future research direction. The problem discussed in the study is related to three research streams in the humanitarian logistics: damage assessment using UAVs; road recovery (cleaning or repairing); and relief distributions. The most relevant studies in each stream and our contributions are discussed in the rest of this section.

\subsection{Damage assessment using UAVs}

The first applications of UAVs go back to the military for inspection and mapping areas. Recently, UAVs are used in commercials (Carlsson \& Song 2017; Agatz et al. 2016), healthcare (Scott \& Scott 2017) and humanitarian logistics. Companies such as Amazon (Amazon 2016), DHL (DHL 2014) and Google (Google 2014) use UAVs for last-mile package delivery in urban areas.

Applications of UAVs in humanitarian logistics involve damage assessment (Huang et al. 2017), locating casualties (Molina et al. 2012; Choi-Fitzpatrick et al. 2016; Zheng et al. 2019), and transporting emergency/medical supplies to affected sites (Nedjati et al. 2016; Chowdhury et al. 2017). According to the 2014 report of the United Nations Office for the Coordination of Humanitarian Affairs (OCHA 2014), UAVs were used for the first time in the 2010 Port-au-Prince Earthquake in Haiti, 2012 Hurricane Sandy in 
Haiti, and 2013 Typhoon Haiyan in the Philippines for real-time data collection and situation monitoring. In the Typhoon Haiyan, UAVs were used to set up a base of operations. This base checked the availability of roads using images that were taken by UAVs. The report clearly mentions that more research is needed to identify the comparative advantages of using UAVs and integrating UAV data collection into the needs of other humanitarian functions. In 2012 Hurricane Sandy in Haiti, International Organization for Migration (IOM) made several flights within a few days over the affected area to assess the destroyed buildings. They compared the real-time processed images with the previously taken ones and cross-checked them to count the collapsed buildings. Those high-definition images enabled them to map the dumps, standing water, road conditions, and areas prone to flood. Otto et al. (2018) provide a comprehensive literature survey on the emerging applications of UAVs. They discuss the UAV applications in the agriculture (e.g., assessing crop health, soil properties, and monitoring livestock), transportation (e.g., package delivery), and disaster management (e.g. recording films and taking pictures of affected sites). They categorize UAVs based on their characteristics (e.g., motion specifics, flight range, and information processing features). They also review some UAV-related optimization papers according to their objective functions, UAV characteristics, and their applications.

The applications of UAVs in humanitarian logistics are mainly studied from technical rather than managerial perspectives. This application is new and has very sparse literature in operations management. Recently, Chowdhury et al. (2017) propose a model to supply emergency commodities to a disaster-affected region via trucks and UAVs as two different transportation modes. In this problem, UAVs are used for the relief 
distribution, not damage assessment. Oruc \& Kara (2018) develop a bi-objective model for routing UAVs and motorcycles in an affected area to provide damage information about its population centers (nodes) and road segments (links). To obtain solutions as fast as possible, a heuristic approach is developed to solve the model. The model and its solution approach are tested for a district in Istanbul city. In contrast to our first model, they do not make any prepositioning decisions in their model. They assume that all UAVs and motorcycles are located at a known depot and UAVs should return to the depot before getting out-of-charge. Also, their problem does not include road recovery and relief distribution components. Balcik (2017) studies a problem similar to Oruc \& Kara (2018) but the focus is on monitoring disaster impacts on population centers (nodes) not roads (links). Similarly, Lim et al. (2018) use UAVs for power network damage assessment. The impact of damage information in the recovery process of the power network and its ultimate performance in servicing customers are not studied in their work.

To the best of our knowledge, this is the first study that develops a comprehensive model for prepositioning and routing of UAVs in the pre-disaster preparedness and investigates its impact on the post-disaster road recovery and relief distribution.

\subsection{Road recovery}

There are some qualitative case studies about road recovery (clearing/repairing) in previous disasters such as Hurricane Katrina (Luther 2008; Mendonca \& Hu 2006). Fetter $\&$ Falasca (2011) point out that there are few quantitative and decision support studies in this domain. Recently, several studies have been done on road recovery and improving accessibility after disasters. The road recovery in our problem includes road selection and 
team allocation in the presence of incomplete information. However, most of the existing studies in road recovery only focus on road selection (do not study the impact of team allocation) in the presence of complete information (Yan \& Shih 2009; Liberatore et al. 2014; Aksu \& Ozdamar 2014; Ozdamar et al. 2014; Sahin et al. 2016). For example, Aksu \& Ozdamar (2014) propose an off-line and deterministic mixed-integer model to select the best-blocked paths that should be recovered by a set of recovery teams. The objective function is to minimize the weighted time needed for reconnecting the network. Their model only selects at most $k$ blocked links at each time step for recovery. They are not concerned with the lengths and recovery times of selected links and team allocation. Sahin et al. (2016) develop a deterministic model to provide relief supplies for a set of critical nodes along a moving path. This path may include blocked links that should be cleaned by a single team. There are only one path and one recovery team in their problem. So, there is no need for team allocation. The problem is off-line with complete information and the objective function is minimizing the total time needed for debris removal. Yan \& Shih (2009) develop a deterministic model with complete information for integrated road recovery and relief distribution. They assume that there are at most two repair points in each blocked road with known locations and repair times. Only one recovery team can be assigned to repair each repair point.

Also, there is another group of researchers in the disaster literature in which all roads have preassigned priorities such as Perrier et al. (2008) and Sahin et al. (2013). Perrier et al. (2008) study the problem of cleaning roads in an urban area with snow plowing equipment and Sahin et al. (2013) consider debris cleaning of a road network in the 
response phase. Our problem differs from these studies because the cleaning priorities of roads are determined by the model and are not exogenously given inputs.

The closest studies to ours are Celik et al. (2016), Kasaei \& Salman (2016) and Akbari \& Salman (2017). Celik et al. (2016) develop a multi-period model for cleaning debris and recovering a road network. Similar to our problem, they assume that there is limited information about the recovery time of the roads. This information is updated over time before making decisions. The main decision is to select the best sequence of roads to clear in each period in a way to make the highest connectivity among demand and supply nodes. They formulate the problem as a partially observable Markov decision process and develop a heuristic approach to find a solution for large-scale road networks. There are several differences between this study and our road recovery model. They did not consider the fact that the cleaning/repair time of each road depends on the number of teams assigned to that road. The objective function at each decision-making time step is making the highest connectivity between the demand and supply nodes. However, the impact of recovery decisions on speeding up the relief transportations between demand and supply nodes is not investigated in their model. We believe that their objective is necessary but not sufficient for disaster response operations. This objective function only creates a connection between demand and supply nodes but does not consider the travel time between these nodes. Minimizing travel/response time is one of the most critical and lifesaving objectives in post-disaster operations. These gaps are covered in our problem. In contrast to ours, their model includes sequential, not time-stamp, data acquisition.

Kasaei \& Salman (2016) propose two deterministic mixed-integer models to determine the moving walk for a recovery team in a road network that is disconnected after 
a disaster. In the first model, the team recovers a subset of blocked roads to reconnect the network in the minimum time. While the second model maximizes the total benefit obtained by reconnecting a subset of network components within a time limit. They show both models are NP-hard and propose a heuristic approach based on variable neighborhood search to obtain near-optimal solutions for large-scale networks in rational computational times. In contrast to our model, they assume that recovery times of blocked links are known in advance (complete information). Also, the models are restricted to a single team and the interdependency between road recovery and relief distribution is ignored in their models. Akbari \& Salman (2017) extend the problem of Kasaei \& Salman (2016) by considering several recovery teams. They develop a deterministic mixed-integer model with complete information to select the best moving walks for each recovery team in a way to reconnect the road network in the least possible time. They simplify the problem by assuming that several teams cannot work simultaneously on a single link to recover it faster. This assumption is relaxed in our model. We assume that several teams can work concurrently on a single link. In this case, the recovery time of the link reduces proportionally.

Our multi-period road recovery model has some features that differentiate it from existing studies: 1) the model is online and the required information (damage/debris levels of blocked roads) is acquired over the time through a model-based approach; 2) there are more than one recovery teams and they are free to work concurrently on the blocked roads if it is necessary. So, the impact of team allocation on the road selection decisions is investigated; and 3) the impact of the road recovery decisions on speeding up the relief distribution operation is investigated. To the best of our knowledge, there is no model with these features in the road recovery literature. 


\subsection{Relief distribution}

The relief distribution has very rich literature in humanitarian logistics. Several researchers review existing models from different perspectives. Luis et al. (2012) classify the disaster relief routing papers according to the characteristics (e.g., vehicle fleet type, allocation policy, and the number of depots) of developed models. Celik (2016) classify the network restoration papers by problem type (e.g., debris collection and disposal, Infrastructure restoration, and network reconstruction) and their solution methods (e.g., exact, heuristic, metaheuristic, and simulation methods). Ozdamar \& Ertem (2015) categorize humanitarian logistics papers in terms of their modeling features and formulation structures (e.g., objective functions, constraints, and solution methods) as well as the utilized technologies to facilitate the implementation of the models. This problem aims to distribute prepositioned relief items to affected people in a fast (Campbell et al. 2008), fair (Tzeng et al. 2007) and least costly (Camacho-Vallejo et al. 2015) way. For example, Salmeron \& Apte (2010) develop a two-stage optimization model for relief distribution in disasters. The first stage of the model determines strategic resources (warehouses, medical facilities, shelters, etc.) expansion decisions in the pre-disaster situation. The second stage deals with the logistics of response operations (delivery of relief commodities and transportation of casualties) in the post-disaster situation. Vanajakumari et al. (2016) develop a bi-objective model to simultaneously 1) locate relief stocks; 2) determine their inventory levels; 3) select size and number of trucks; and 4) route the trucks from stocks to demand points. The objective functions are reducing the response time and minimizing the logistics cost. Most of these studies either ignore the road disruption in transportation networks or they assume that a subset of roads is disrupted and cannot be 
used in the relief distribution. They ignore the recovery possibility of these roads during the response operation.

Some studies consider the relief distribution and road recovery simultaneously (Yan \& Shih 2009; Kibar \& Salman 2013; Nurre \& Sharkey 2014). Yan \& Shih (2009) propose a multi-objective and multi-commodity network flow model on a time-space network to schedule road recovery and relief distribution. The road recovery part of the model only makes road selection decisions and recovery times of all roads are known in advance and equal. In contrast to our model, only one team can be assigned to each road and the objective function is maximizing the connectivity between demand and supply nodes. They consider two types of blocked roads in their problem. Prioritized roads should be recovered before a specific time due to their importance. Similarly, Kibar \& Salman (2013) and Nurre \& Sharkey (2014) assume that the precise recovery times of all blocked roads are known at the beginning. In contrast to ours, their models are not online. These features differentiate their problems from ours.

The main contributions of this study to the short-term road recovery literature are four-fold:

- Developing an integrative framework: To the best of our knowledge, our study is the first one that simultaneously considers 1) damage assessment, 2) road recovery, and 3) relief distribution. This integration makes it possible to analyze how the decisions of each model affect the outcomes of the others.

- Handling incomplete information: This is the first study that handles incomplete information in road restoration operations using a novel model-based data diffusion approach. 
- Considering pre-disaster UAVs preparedness for damage assessment: In this study, we develop a comprehensive model for prepositioning UAVs in the pre-disaster preparedness and investigate its impact on the post-disaster response (road recovery and relief distribution) operations.

- Optimizing the recovery team allocation in the road recovery operation: There are several recovery teams in our problem, and they are free to work concurrently on blocked roads if it is necessary. We investigate the impact of team allocation on the road selection decisions. 


\section{Chapter 3}

\section{MODEL FORMULATION AND SOLUTION APPROACH}

Consider an urban area predicted to be stricken by a disaster in the near future. The road infrastructure of the area is represented by an undirected network. In the preparedness phase, some UAVs are prepositioned over the network (at nodes or links) to facilitate road inspection and damage assessment after the disaster. The following questions should be answered in the preparedness phase: 1) how many UAVs should be prepositioned in the network? 2) where are the best places to locate UAVs? 3) how many charging stations are needed to recharge the UAVs during the assessment process? 4) where are the best places to locate charging stations? and 5) what are the best moving walks for located UAVs? These decisions are made in a way to minimize the total preparedness cost (the deployment cost of UAVs, the locating cost of charging stations and the operational cost of scanning roads) and the scan completion time of the whole network.

After the disaster and in the response phase, the located UAVs scan the road network according to the walks determined in the preparedness phase. They gradually scan the network and send pictures to a ground control center for damage assessment. The UAVs are remotely piloted and are able to take close pictures of the blocked roads. These pictures provide precise estimations of damage/debris levels and required recovery (repair or cleaning) times. The damage/debris information of the blocked roads is periodically sent to recovery teams that are retrieving the road network. The prepositioning decisions of UAVs determine how much damage/debris information is available to be sent to the recovery teams at each period (multi-size and time stamp data diffusion). 
After receiving the first data batch, the recovery teams make the following decisions: 1) which subset of scanned and blocked roads should be selected for recovery? and 2) how many recovery teams should be assigned to each selected road? After implementing the first set of recovery decisions, the data of scanned roads are updated, and the next set of recovery decisions is made. This means that road recovery is online and updated based on the damage assessment information. Recovery decisions are made in a way to accelerate the distribution of relief supplies (commodities, casualties, and services) between demand and supply nodes.

The model of prepositioning UAVs in the preparedness phase to diffuse damage/debris data in the response phase is discussed in next two sections addresses the problems of relief distribution and road recovery in the response phase. The notation used in this study is summarized in Table 1A (see Appendix A).

\subsection{Model for prepositioning damage assessment facilities}

Some disasters (such as floods, hurricanes, and tornados) have an advance warning and initial reports issued by the National Weather Center contain an accurate prediction of the disaster's projected trajectory and its target region (NHC 2018). In the preparedness phase that we want to preposition UAVs, we know that the disaster may hit somewhere inside the predicted target region. However, the exact location of the stricken area is unknown. In no-warning disasters (such as earthquakes), the target region is the whole disaster-prone area that should always be prepared against disasters. Following covering models that are broadly used to locate emergency service facilities (Farahani et al. 2012), we preposition UAVs and their charging stations in a way to cover the whole target region. 
Covering the whole target region makes preparedness against all scenarios that can be defined for the location of the stricken parts inside the target region.

Let $G=(N, L)$ be an undirected network representing the road network of the target region threatened by a disaster. To get prepared for the disaster, some UAVs and their charging stations are prepositioned to cover the whole network. These facilities will be used after the disaster to scan the road network and diffuse the damage/debris data. The following decisions should be made for prepositioning UAVs: how many charging stations should be prepositioned in the network? Where are the best places to locate the charging stations? How many UAVs should be kept at each station? What is the moving walk for each UAV (a walk starts from the original charging station of that UAV, passes through several connected roads to scan them, goes through some intermediate stations for recharging and ends at a charging station)? Some constraints should be considered in the decision-making process. The fly distance of the UAVs depends on their battery capacity and is limited. So, during their walks, the UAVs should reach a charging station before becoming discharged. The scan completion time is the moment in which the scan process of the road network terminates. All roads of the network should be scanned by at least one UAV before the scan completion time. Scanning a road happens when the first UAV passes through that road. The decisions are made in a way to minimize the scan completion time and minimize the total preparedness cost for scanning the road network. The preparedness cost includes the locating cost of charging stations, the deployment cost of UAVs, and the operational cost of moving UAVs through the roads.

To control the moving distance of UAVs in the network, we suggest a simple network reformation: First, a new distance unit, called NDU hereafter, is defined. NDU is 
the longest distance that the length of all roads and the fly distance of UAVs can be estimated as coefficients of NDU. Then, the length of all roads in the network is unified by adding some auxiliary nodes. After adding auxiliary nodes, the length of all roads would be one NDU. After unification, it would be much easier to control the travel distance of UAVs in the network (there is no need to define binary variables to determine the travel sequence of roads in the walks). Figure 5a shows a sample road network. The lengths of links $\left(n_{1}, n_{2}\right),\left(n_{3}, n_{4}\right)$, and $\left(n_{4}, n_{5}\right)$ are one NDU. So, there is no need to add auxiliary nodes to these links. The lengths of links $\left(n_{1}, n_{5}\right),\left(n_{2}, n_{3}\right)$, and $\left(n_{3}, n_{5}\right)$ are two NDUs. Therefore, an auxiliary node should be inserted to the middle of these links (nodes $n_{6}, n_{7}$ and $\left.n_{8}\right)$. Similarly, the length of the link $\left(n_{2}, n_{5}\right)$ is three NDUs. So, two auxiliary nodes should be located over the link in a way to make sure that the lengths of three newly generated links would be one NDU (nodes $n_{9}$ and $n_{10}$ ). Figure $5 \mathrm{~b}$ shows the unified network resulted after the network reformation, $G^{\prime}=(\hat{N}, L)(|\hat{N}| \geq|N|$ and $|\dot{L}| \geq|L|)$.
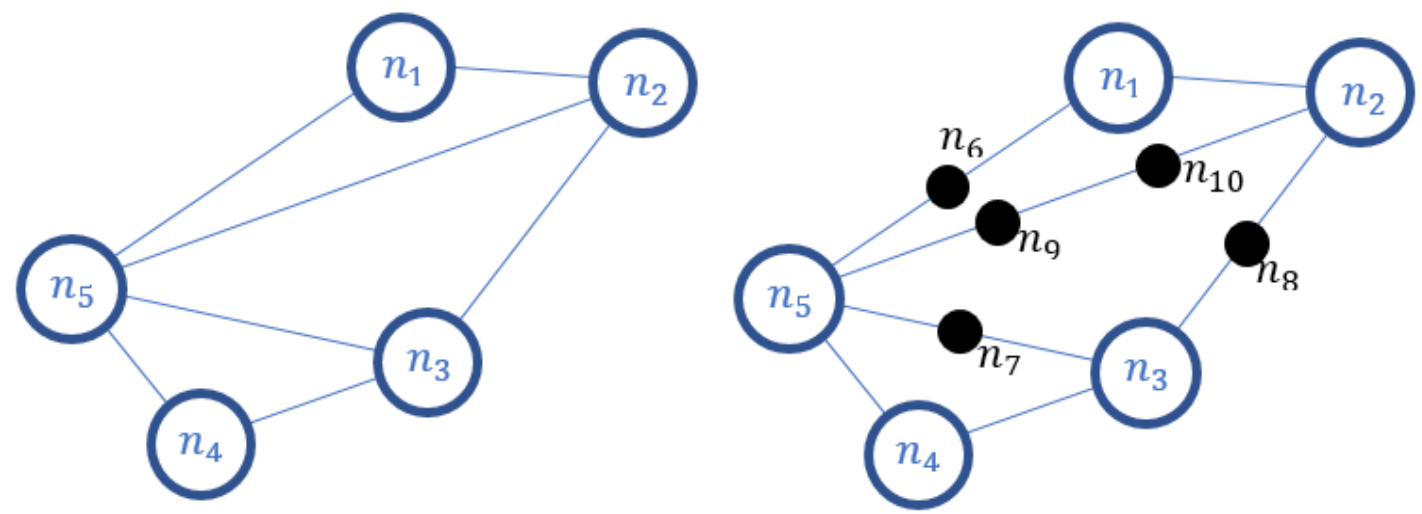

Figure 5. Network reformation for a sample road network. (a) The original network. (b) The Unified Network. 
Considering that UAVs move through the network with the speed of $v$ (NDU per time unit), the time needed to travel a link in the unified network would be $1 / v$. So, road selection decisions are made at discrete times with the subsequent difference of $1 / v$ which is shown by set $T=\{t\}(|T| \geq|L|)$. Model P1 optimizes prepositioning decisions for UAVs in a unified road network:

$$
\begin{aligned}
& \mathrm{P} 1: \quad \mathrm{MIN} \quad Z_{1}=\sum_{n=1}^{|\hat{N}|} f_{n} \cdot Z_{n}+\sum_{n=1}^{|\hat{N}|} p_{n} \cdot x_{n}+\sum_{t=1}^{|T|} \sum_{n=1}^{|\hat{N}|} \sum_{\substack{m=1 \\
m \neq n}}^{|\hat{N}|} \text { o. } y_{(\underset{n, m}{t})}^{t} \\
& \text { MIN } Z_{2}=\Psi \\
& \text { S.T. } \quad x_{n} \leq M . z_{n} \quad(\forall n \in N) \\
& \sum_{m=1}^{|N|} y_{(\overrightarrow{n, m})}^{1} \leq x_{n} \quad(\forall n \in N) \\
& \sum_{n=1}^{|\hat{N}|} y_{(\underset{n, m}{t})}^{t}=\sum_{k=1}^{|\hat{N}|} y_{(\underset{m, k}{t+1})}^{t+} \quad(\forall t=1,2, \ldots,|T|-1)(\forall m \in \hat{N}) \\
& \sum_{n=1}^{|\hat{N}|} y_{(\overrightarrow{n, m})}^{t} \leq M \cdot z_{m} \quad(\forall t=d, 2 d, 3 d, \ldots, \zeta d)(\forall m \in \hat{N})(6)
\end{aligned}
$$

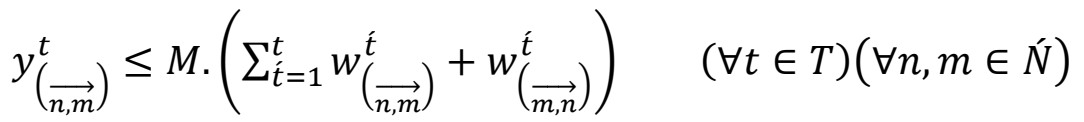

$$
\begin{aligned}
& \left.w_{(\overrightarrow{n, m})}^{t} \leq y_{(\overrightarrow{n, m}}^{t}\right) \quad(\forall t \in T)(\forall n, m \in \hat{N}) \\
& \sum_{t=1}^{|T|} w_{(\overrightarrow{n, m})}^{t}+w_{(\overrightarrow{m, n})}^{t}=a_{(n, m)} \quad(\forall n, m \in \hat{N}) \\
& y_{(\overrightarrow{n, m})}^{t} \leq M \cdot a_{(n, m)} \quad(\forall t \in T)(\forall n, m \in N) \\
& \Psi \geq \sum_{t=1}^{|T|} t \cdot w_{(\overrightarrow{n, m})}^{t} \\
& z_{n} \text { and } w_{(\overrightarrow{n, m})}^{t} \in\{0,1\} \quad(\forall t \in T)(\forall n, m \in \hat{N}) \\
& x_{n} \text { and } y_{(\underset{n, m}{t})}^{t} \geq 0 \quad(\forall t \in T)\left(\forall n, m \in N^{\prime}\right)
\end{aligned}
$$

The objective function (1) minimizes the total preparedness cost for scanning the road network. The first, second and third terms in (1) represent the locating cost of charging stations, the deployment cost of UAVs, and the operational cost of moving UAVs through the roads. The objective function (2) minimizes the scan completion time of the whole 
network. Constraint (3) ensures that UAVs are initially located at nodes where there is a station. Based on the constraint (4), the total number of UAVs leaving a station in the first time unit is less than or equal to the number of UAVs prepositioned at that station. At other intermediate times, the total number of UAVs entering into a node should be equal to the number of UAVs leaving or staying at that node (constraint (5)). The UAVs have limited flying time and should be recharged after traversing $d$ (NDU). According to the constraint (6), the UAVs should pass through a charging station before becoming out-of-charge ( $\zeta$ represents the largest integer value that $\zeta d \leq|T|$ ). The necessity of recharging may make UAVs traverse a road (link) more than one time. Constraints (7) and (8) ensure that a road will be scanned for the first time a UAV passes through that road. Constraint (9) determines the nodes of the network that are connected by a road and should be scanned by a UAV in either direction. Similarly, constraint (10) ensures that the UAVs can move between a pair of nodes if they are connected by a road. Constraint (11) makes sure that the scan completion time of the network is greater than or equal to the scan times of all roads. Model P1 is a combination of a node- and arc-based formulations. While UAVs and their charging stations are located at nodes, UAVs are moving through the links/roads for scanning and should cover all of them before the scan competition time.

The decisions made in this operation (Model 1) determine when each road will be scanned by the UAVs and its damage/debris information will be available. This model controls the data diffusion speed in the integrative framework and constitutes its modelbased data diffusion part. The damage/debris data of the scanned roads will be gathered and periodically shared with Model 2. 


\subsection{Solution approach}

Model P1 is mixed-integer linear programming with $|\hat{N}|+|\hat{N}|^{2} .|T|$ binary variables. This high computational complexity makes Model P1 very time-consuming or even unsolvable for large real-size road networks. In this section, a heuristic approach is developed to solve the model and find close-to-optimal solutions for large-scale networks. Detailed investigation of Model P1 reveals some characteristics for the optimal solution that will be used in developing the heuristic approach (refer to Appendix B for proofs).

Proposition 1: For a given number of UAVs $\left(\sum_{n=1}^{|\hat{N}|} x_{n}=n^{U}\right)$, the minimum $\Psi$ needed to scan the whole network, $\Psi^{\operatorname{Min}}\left(n^{U}\right)$, is $\left\lceil\frac{|L|}{n^{U}}\right\rceil$.

This proposition determines the relationship between the number of UAVs and the scan completion time in the network. The next proposition shows the relationship between $\mathrm{n}^{\mathrm{U}}, \Psi^{\operatorname{Min}}\left(\mathrm{n}^{\mathrm{U}}\right)$ and the number of charging stations $\left(\sum_{\mathrm{n}=1}^{|\hat{N}|} \mathrm{z}_{\mathrm{n}}=\mathrm{n}^{\mathrm{S}}\right)$.

Proposition 2: For a given number of $U A V s\left(n^{U}\right)$ and its minimum scan completion time $\left(\Psi^{\operatorname{Min}}\left(n^{U}\right)\right)$, the number of charging stations that should be located in the network is $n^{S} \leq$ $\left\lceil\frac{\mid L ́}{\operatorname{Min}\left\{2 \Psi^{\operatorname{Min}}\left(n^{U}\right), d\right\}}\right]$. Finding a close form equation for $n^{S}$ is impossible because it completely depends on the topology of the network. Model P2 determines the minimum $n^{S}$ for the network:

P2: $\quad \operatorname{MIN} \quad Z_{3}\left(n^{U}, \Psi^{\operatorname{Min}}\left(n^{U}\right)\right)=\sum_{n=1}^{|\hat{N}|} z_{n}$

S.T. $\quad \sum_{n=1}^{|\hat{N}|} x_{n} \leq n^{U}$

$x_{n} \leq M \cdot z_{n}$

$(\forall n \in N)$ 


$$
\begin{aligned}
& \sum_{m=1}^{|N|} y_{(\underset{n, m}{1})}^{1} \leq x_{n} \\
& (\forall n \in N) \\
& \sum_{n=1}^{|\hat{N}|} y_{(\underset{n, m}{t})}^{t}=\sum_{k=1}^{|\hat{N}|} y_{(\underset{m, k}{t+1})}^{t+}\left(\forall t=1,2, \ldots, \operatorname{Min}\left\{2 \Psi^{\operatorname{Min}}\left(n^{U}\right), d\right\}-1\right)(\forall m \in \hat{N})(18) \\
& \sum_{n=1}^{|N|} y_{(\overrightarrow{n, m})}^{t} \leq M \cdot z_{m} \quad\left(t=\operatorname{Min}\left\{2 \Psi^{\operatorname{Min}}\left(n^{U}\right), d\right\}\right)(\forall m \in N) \\
& y_{(\overrightarrow{n, m})}^{t} \leq a_{(n, m)} \quad\left(\forall t=1,2, \ldots, \operatorname{Min}\left\{2 \Psi^{\operatorname{Min}}\left(n^{U}\right), d\right\}\right)(\forall n, m \in N) \\
& \sum_{t=1}^{\operatorname{Min}\left\{2 \Psi^{\operatorname{Min}}\left(n^{U}\right), d\right\}} y_{(\overrightarrow{n, m})}^{t}+y_{(\overrightarrow{m, n})}^{t} \geq a_{(n, m)} \quad(\forall n, m \in \hat{N}) \\
& z_{n} \in\{0,1\} \\
& x_{n} \text { and } y_{(\underset{n, m}{t})} \geq 0 \\
& (\forall n \in N) \\
& (\forall t \in T)(\forall n, m \in N)
\end{aligned}
$$

The value of $\sum_{\mathrm{n}=1}^{|\hat{N}|} \mathrm{z}_{\mathrm{n}}^{*}$ represents the minimum number of charging stations that should be located in the road network with $\mathrm{n}^{\mathrm{U}} \mathrm{UAV}$ s to scan the whole network in the minimum possible $\Psi\left(\Psi^{\operatorname{Min}}\left(n^{U}\right)\right)$. The computational complexity of Model P2 is much less than Model P1.

These results are used to develop a heuristic approach. This approach first locates the minimum number of charging stations, then prepositions the minimum number of UAVs and finally determines their moving walks throughout the network. The steps of the heuristic approach are as follows:

Step 1: For a predetermined $\dot{\Psi}$, Model P2 with $n^{U}=M$ ( $M$ is a very large constant value $)$ and $\Psi^{\operatorname{Min}}\left(n^{U}\right)=\dot{\Psi}$ is used to determine the locations of charging stations in the unified network. After locating the charging stations, the rest of the nodes that do not encompass a charging station and their connecting links are removed from the network. Any pair of the remaining nodes can be connected by a new link if a moving path with the length of less than or equal to $\operatorname{Min}\{2 \dot{\Psi}, d\}$ exists between them in the unified network. Enough new 
links should be defined in a way to cover all the links in the unified network. The number of nodes and links in the newly constructed network, called super-network hereafter $\left(G^{\prime \prime}=\right.$ $\left.\left(N^{\prime \prime}, L^{\prime \prime}\right)\right)$, is much less than the unified network $(G)$. This significantly reduces the computational complexity of the model developed in the next step. A sample unified network and its corresponding super-network are shown in Figure 6. Parameters $\dot{a}_{(n, m)}$ and $\bar{d}_{(n, m)}$ show the number of links and the average distance between nodes $n$ and $m$ in the super-network, respectively.
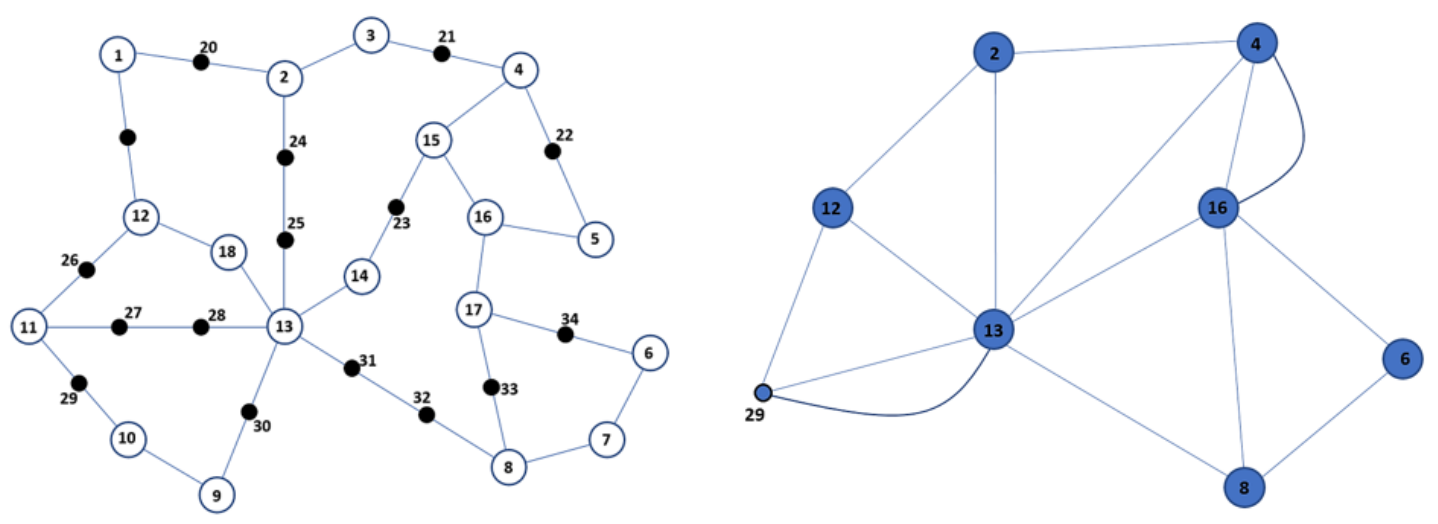

Figure 6. The super-network constructed for a sample unified network with $d=4$ (NDU) and $\Psi=12$ (NDU). (a) A sample unified network $\left(\left|N^{\prime}\right|=35\right)$. (b) The constructed super-network $\left(\left|N^{\prime \prime}\right|=8\right)$.

Step 2: Based on the super-network developed in Step 1, we use Model P3 to preposition UAVs and determine their moving walks through the network:

P3: $\quad \operatorname{MIN} \quad Z_{4}(\dot{\Psi})=\sum_{n=1}^{\left|N^{\prime \prime}\right|} p_{n} \cdot x_{n}+\sum_{t=1}^{\left|\frac{\dot{\Psi}}{\operatorname{Min}\{2 \dot{\Psi}, d\}}\right|} \sum_{n=1}^{\left|N^{\prime \prime}\right|} \sum_{\substack{m=1 \\ m \neq n}}^{\left|N^{\prime \prime}\right|} o \cdot \bar{d}_{(n, m)} \cdot y_{(\underset{n, m}{t})}$

$$
\begin{aligned}
& \text { S.T. } \quad \sum_{m=1}^{\left|N^{\prime \prime}\right|} y_{(\underset{n, m}{1})}^{1} \leq x_{n} \quad\left(\forall n \in N^{\prime \prime}\right) \\
& \left.\sum_{n=1}^{\left|N^{\prime \prime}\right|} y_{(\underset{n, m}{t})}^{t}=\sum_{k=1}^{\left|N^{\prime \prime}\right|} y_{(\underset{m, k}{t+1})}^{t}\left(\forall t=1,2, \ldots, \mid \frac{\dot{\psi}}{\operatorname{Min}\{2 \dot{\Psi}, d\}}\right\rfloor-1\right)\left(\forall m \in N^{\prime \prime}\right) \\
& \sum_{t=1}^{\left\lfloor\frac{\dot{\Psi}}{\operatorname{Min}\{2 \dot{\Psi}, d\}}\right\rfloor} y_{(\underset{n, m}{t})}^{t}+y_{(\underset{m, n}{t})}^{t}=\dot{a}_{(n, m)} \quad\left(\forall n, m \in N^{\prime \prime}\right)
\end{aligned}
$$




$$
\begin{array}{lc}
y_{(\overrightarrow{n, m})}^{t} \leq \dot{a}_{(n, m)} & \left.\left(\forall t=1,2, \ldots, \mid \frac{\dot{\psi}}{\operatorname{Min}\{2 \dot{\Psi}, d\}}\right\rfloor\right)\left(\forall n, m \in N^{\prime \prime}\right) \\
y_{(\overrightarrow{n, m})}^{t} \in\{0,1\} & \left(\forall t=1,2, \ldots,\left|\frac{\dot{\Psi}}{\operatorname{Min}\{2 \dot{\Psi}, d\}}\right|\right)\left(\forall n, m \in N^{\prime \prime}\right) \\
x_{n} \geq 0 & \left(\forall n \in N^{\prime \prime}\right)
\end{array}
$$

The objective function (24) minimizes the sum of the prepositioned UAVs' deployment cost (the first term) and the operational cost of moving UAVs through the roads (the second term). Since the scan completion time is $\dot{\Psi}$, the maximum number of links that can be traversed by a UAV is $\left\lfloor\frac{\dot{\Psi}}{\operatorname{Min}\{2 \dot{\Psi}, d\}}\right\rfloor$. At each time unit, one link is traversed by a UAV. According to the constraint (25), the total number of UAVs leaving a station in the first time unit should be less than or equal to the number of UAVs prepositioned at that station. At the intermediate times, the total number of UAVs entering a station should be equal to the total number of UAVs leaving or staying at that station (constraint 26). Constraint (27) ensures that all the links of the super-network (and consequently all the links of the original network) would be scanned by the UAVs. Based on the constraint (28), the UAVs can only move from node $n\left(\forall n \in N^{\prime \prime}\right)$ to node $m\left(\forall m \in N^{\prime \prime} \mid n \neq m\right)$ if a link exists between these nodes.

Solving Models P2 (with $n^{U}=M$ and, $\Psi^{\operatorname{Min}}\left(n^{U}\right)=\dot{\Psi}$ ) and P3 for the unified and super networks determines the number of charging stations, number of UAVs and their walks (and the associated total preparedness cost) for the road network with the predetermined $\dot{\Psi}$. Repeating these steps for different values of $\dot{\Psi}$ provides all the Pareto optimal solutions for Model P1. In this model, all the roads of the network have similar priorities for damage assessment. Sometimes, some roads of the network are more prone to be damaged in disasters. Roads located beside oceans, seas, rivers, or epicenters or roads 
ending at the population centers located in highly disaster-prone regions have higher probabilities to be damaged by disasters. In Appendix C, we propose a new model for prepositioning damage assessment facilities in a network with prioritized roads.

\subsection{Performance of the heuristic approach}

Without loss of generality, we focus on hurricanes in our case problem as weatherrelated, rapid-onset natural disasters threatening major parts of coastal regions in the US. Suppose that a hurricane has formed in the Atlantic Ocean and is moving toward the southeast coast of the US according to the forecast cone of the National Weather Service. The southeast coast is the most hurricane-prone part of the US. According to the statistics of the National Oceanic and Atmospheric Administration (NOAA), 2.2 major hurricanes hit this area on average each year (NHC 2012). The hurricane's forecast cone that consists of several target circles represents its probable track. Suppose that the area inside the dashed circle in Figure 7a is located inside the hurricane's forecast cone and should be prepared for the disaster by prepositioning UAVs and their charging stations. This area includes the whole Miami-Dade County and the southern part of Broward County (Miramar, Davie, Weston, Hollywood, Pembroke Pines, and Fort Lauderdale suburban). The road network of this area is represented in Figure 7b. Only highways and main roads that have critical roles in relief distribution are considered in the road network. The length of the roads/links in the network is equal to the driving distance determined by Google Maps. The NDU that is used to unify the road network is $1 \mathrm{mi}$. Federal Aviation Administration (FAA) authorized all commercial and humanitarian UAVs to be flown below $200 \mathrm{ft}$. A UAV flying in $200 \mathrm{ft}$. can monitor a circle of at most $130 \mathrm{ft}$. radius (Oruc 
\& Kara, 2018). Since the distance between roads in our network is at least $1 \mathrm{mi}$., it is not possible that more than one road segment can be scanned by each UAV. The deployment cost of a UAV and its operational cost per NDU is $\$ 500$ and $\$ 5$, respectively. The cost of locating a charging station is $\$ 2000$. Considering the average performance of UAVs exist in the market, we assume that the UAVs scan the road network with an average speed of $20 \mathrm{mi}$ per hr. A fully-charged UAV can travel 6 NDU throughout the network before becoming out of charge. Considering the time needed to recharge UAVs, they can scan the road network for at most $8 \mathrm{hr}$. per day. We solved Model P1 and the heuristic approach for 6 sub-networks of the case network for the scan completion time of $\Psi=12$ (Model P1 could not find the optimal solution for the case network in 72 hours).
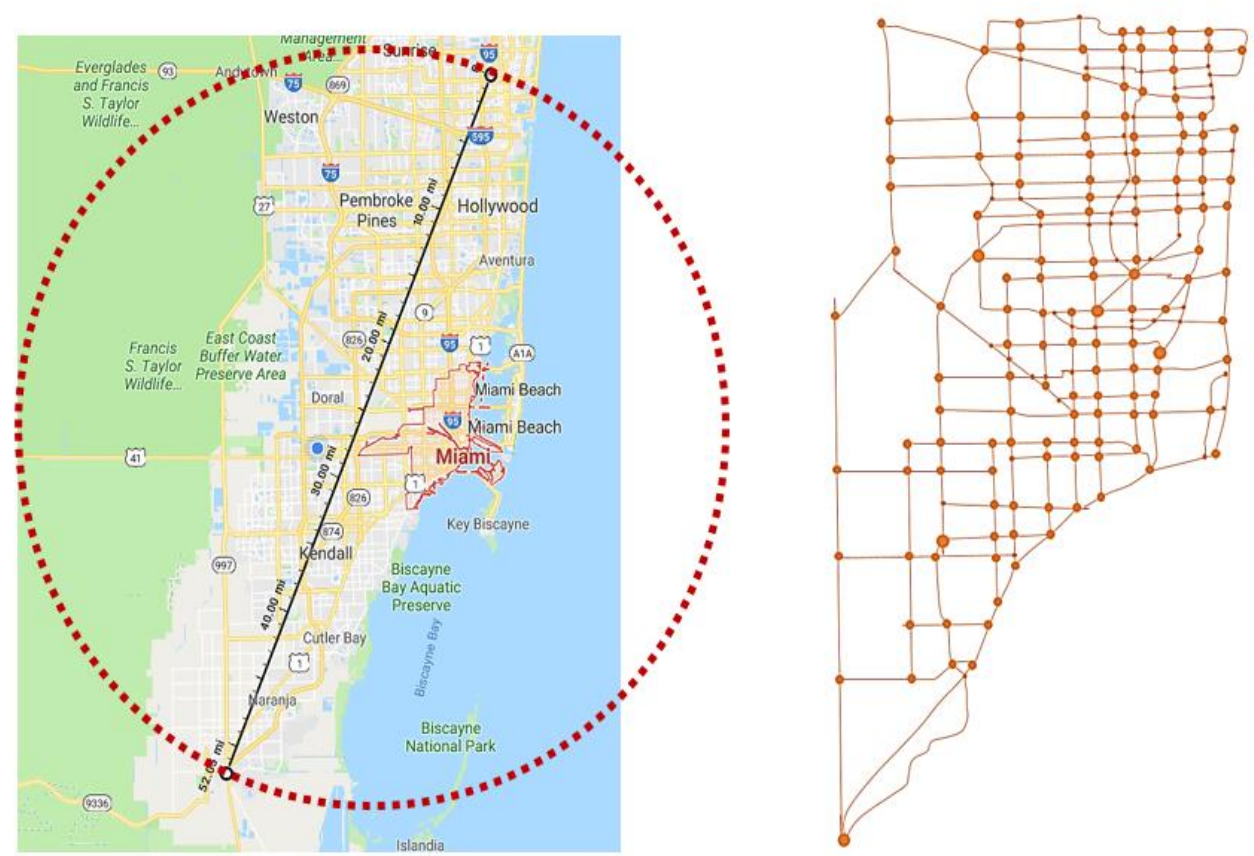

Figure 7. The case problem. (a) The target area. (b) The road network of the target area. 
The computational time of the heuristic approach for the case network and its subnetworks was less than a minute, much lower than the computation times of Model P1. Model P1 was solved using CPLEX software on a Dell computer with Windows 10, an Intel $i 7$ processor, and $125 \mathrm{~GB}$ of installed RAM. Table 1 shows that the performance of the heuristic approach is acceptably high and the optimality gap between the solution of the heuristic approach and the optimal solution of Model P1 has never been more than $3.8 \%$.

Table 1. The accuracy of the solutions provided by the heuristic approach.

\begin{tabular}{|c|c|c|c|c|c|c|}
\hline Sub-networks & $\begin{array}{c}\text { No. of nodes } \\
\text { in the original } \\
\text { sub-network }\end{array}$ & $\begin{array}{c}\text { No. of nodes } \\
\text { in the unified } \\
\text { sub-network }\end{array}$ & $\begin{array}{c}\text { The cost of } \\
\text { Model P1 }\end{array}$ & $\begin{array}{c}\text { The cost of } \\
\text { the heuristic }\end{array}$ & $\begin{array}{c}\text { Solution } \\
\text { time for } \\
\text { Model P1 }\end{array}$ & difference \\
\hline $\begin{array}{c}\text { Sub-network } \\
1\end{array}$ & 11 & 65 & $\$ 23,865$ & $\$ 24,440$ & $00: 13: 45$ & $2.4 \%$ \\
\hline $\begin{array}{c}\text { Sub-network } \\
2\end{array}$ & 20 & 116 & $\$ 41,205$ & $\$ 42,800$ & $00: 55: 17$ & $3.8 \%$ \\
\hline $\begin{array}{c}\text { Sub-network } \\
3\end{array}$ & 25 & 143 & $\$ 50,405$ & $\$ 51,463$ & $01: 17: 56$ & $2.1 \%$ \\
\hline $\begin{array}{c}\text { Sub-network } \\
4\end{array}$ & 36 & 195 & $\$ 66,700$ & $\$ 69,057$ & $05: 26: 10$ & $3.5 \%$ \\
\hline $\begin{array}{c}\text { Sub-network } \\
5\end{array}$ & 42 & 233 & $\$ 79,360$ & $\$ 81,502$ & $10: 11: 48$ & $2.7 \%$ \\
\hline $\begin{array}{c}\text { Sub-network } \\
6\end{array}$ & 53 & 265 & $\$ 91,170$ & $\$ 93,530$ & $25: 07: 33$ & $2.5 \%$ \\
\hline
\end{tabular}

The heuristic approach was used to preposition UAVs in the case network. Figure 8 shows the tradeoff between $\Psi$ and the pre-disaster preparedness cost in the case network. As seen in the figure, reducing $\Psi$ makes a concave-up increment in the cost. This means that accelerating the data acquisition in the post-disaster response phase increasingly boosts the preparedness cost of locating monitoring facilities in the pre-disaster preparedness phase. 


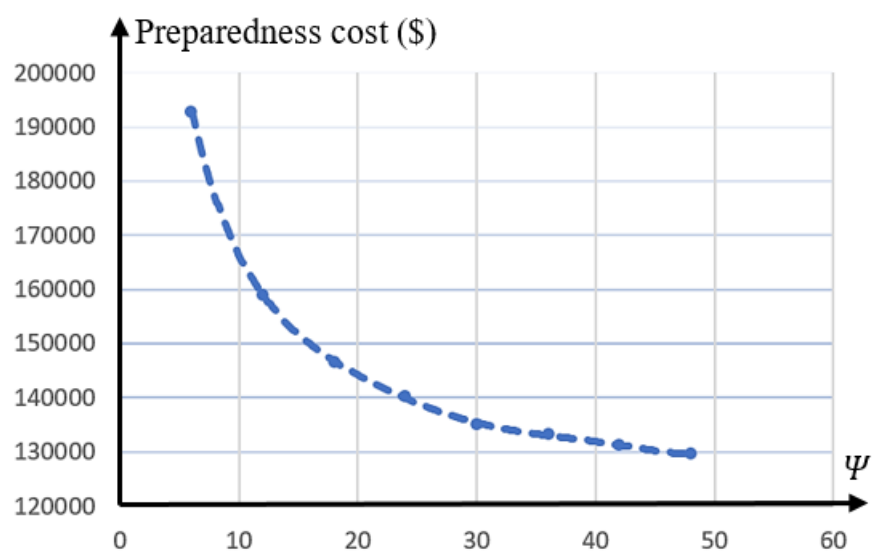

Figure 8 . The tradeoff between $\Psi$ and the pre-disaster cost in the case network.

\subsection{Models for relief distribution and road recovery}

The moving walks determined for UAVs determine when each road of the network will be scanned, and its damage/debris information will be available for road recovery (in Model 2). In this study, we assume that UAVs scan the area according to the moving walks determined before the disaster. In the chaotic post-disaster circumstances and under time pressure, it is not easy to run another model and adjust moving walks for UAVs. Also, the fixed locations of charging stations that are located before the disaster significantly limit the adjustability of moving walks for UAVs after the disaster.

Locating more UAVs in Model 1 augments the preparedness cost, but it reduces the scan completion time and increases the damage/debris data diffusion speed to Model 2. In the next section, we explain the relief distribution operation when no road recovery happens during the disaster response phase. Then, we consider the road recovery possibility in its following Section. We explain how road recovery operations should be scheduled to make the highest acceleration in the relief distribution. 


\subsection{Relief distribution without road recovery}

After disasters, there is an urgent need for relief distribution (goods, casualties, rescue teams, medical personnel, etc.) between a set of supply nodes (such as stocks of prepositioned goods and airports) and demand nodes (such as affected sites and hospitals). Set $P=\{p=(n, m) \mid n, m \in N\}$ includes all pairs of demand and supply nodes that appear after the disaster. The total amount of shipment between the nodes of pair $p \in P$ is $\Theta^{p}$ and the capacity of the transportation fleet assigned to pair $p$ is $\vartheta^{p}$. Usually $\vartheta^{p}<\Theta^{p}$ due to resource scarcity after disasters and the shipments are accomplished through several deliveries. The cost of fulfilling a unit of demand at the demand node of pair $p$ at time $t$ is $c^{p}(t) \cdot c^{p}(t)$ includes the transportation cost and the delay penalty of fulfilling the demand that is materialized at time 0 (the occurrence moment of the disaster). Thus, $c^{p}(t)$ is an increasing function of $t$. Binary parameters of $\gamma_{n}^{p}$ and $\dot{\gamma}_{n}^{p}$ are 1 for node $n \in N$ if it is the supply and demand node of pair $p$, and 0 otherwise. According to the undisrupted links available in the network right after the disaster (determined immediately using satellite images), the shortest transportation time between the nodes of pair $p, \theta^{p}$, is determined by Model P4. In Model P4, binary parameter $\gamma_{(n, m)}$ is 1 if the link $(n, m)$ is undisrupted and can be used by the transportation fleet, and 0 otherwise.

$$
\begin{aligned}
& \text { P4: } \text { MIN } \quad Z_{6}(p)=\theta^{p} \\
& \text { S.T. } \quad \sum_{m=1}^{|N|} \dot{y}_{(\overrightarrow{n, m})}^{1} \leq \dot{x}_{n} \quad(\forall n \in N) \\
& \left.\sum_{n=1}^{|\hat{N}|} \hat{y}_{(\overrightarrow{n, m})}^{t}=\sum_{k=1}^{|\hat{N}|} \hat{y}_{(\overrightarrow{m, k}}^{t+1}\right) \quad(\forall t=1, \ldots,|\hat{T}|-1)(\forall m \in \hat{N}) \\
& \sum_{n=1}^{|\hat{N}|} \dot{y}_{(\overrightarrow{n, m})}^{t}=\dot{\gamma}_{m}^{p} \cdot \vartheta^{p} \quad(t=|\hat{T}|)(\forall m \in \hat{N}) \\
& \dot{x}_{n} \leq \gamma_{n}^{p} \cdot \vartheta^{p} \quad(\forall n \in N)
\end{aligned}
$$




$$
\begin{array}{ll}
\dot{y}_{(\overrightarrow{n, m})}^{t} \leq M \cdot a_{(n, m)} \cdot \gamma_{(n, m)} & (\forall t \in \hat{T})(\forall n, m \in \hat{N}) \\
\dot{y}_{(\overrightarrow{n, m})}^{t} \leq M \cdot \dot{w}_{(\overrightarrow{n, m})}^{t} & (\forall t \in \hat{T})(\forall n, m \in \hat{N}) \\
\left.\theta^{p} \geq t \cdot \dot{w}_{(\overrightarrow{n, m}}^{t}\right) \mid n \neq m & \left(\forall t \in T^{\prime}\right)(\forall n, m \in \hat{N}) \\
\dot{x}_{n} \text { and } \dot{y}_{(\overrightarrow{n, m})}^{t} \geq 0 & (\forall t \in \hat{T})(\forall n, m \in \hat{N}) \\
\dot{w}_{(\overrightarrow{n, m})}^{t} \in\{0.1\} & (\forall t \in \hat{T})(\forall n, m \in \hat{N})
\end{array}
$$

The objective function (31) minimizes the total transportation time needed to accomplish a delivery between the nodes of pair $p(\forall p \in P)$. Constraints (32) and (34) ensure that the relief flow only originates from the supply node of pair $p$ and ends at its demand node, respectively. At the intermediate nodes, the total inflow should be equal to the total outflow (constraint (33)). The outflow from the supply node cannot be more than the transportation capacity (constraint (35)). According to the constraint (36), only undisrupted roads can be used in the delivery process. Constraints (37) and (38) preserve that the completion time of the delivery is greater than or equal to the times at which the vehicles traverse the links of the movement path selected between the supply and demand nodes. Using the shortest transportation time, $\theta^{p *}(\forall p \in P)$, determined for each pair by solving Model P4, the total response cost of satisfying demands in the network would be as follows:

Proposition 3: If disrupted roads of the network will not be recovered during the response phase of the disaster, the total response cost in the relief distribution operation would be $\sum_{p \in P} \sum_{i=1}^{\left[\frac{\theta^{p}}{\vartheta^{p}}\right]} \vartheta^{p} \cdot c^{p}\left(\theta^{p *}+2 \theta^{p *}(i-1)\right)$. 


\subsection{Relief distribution with road recovery}

After the disaster (occurs at time 0), the prepositioned UAVs start scanning the road network according to their walks. They provide precise pictures of disrupted roads located on their walks. The pictures are used to accurately estimate the damage/debris level and the required recovery time of roads. The damage/debris data is accumulated and periodically sent to the recovery teams. Suppose that the first round of data is provided at time $t$ for the recovery teams. Set $L^{(t)}$ includes the disrupted roads that are scanned by the UAVs up to time $t$. The required recovery time for link $l \in L^{(t)}$ if it is recovered by a single team is $r^{l}$ (by increasing the number of recovery teams assigned to this link, its recovery time reduces proportionally). A subset of $L^{(t)}$ is selected by teams for recovery and the recovery teams that are available at time $t, \Lambda^{(t)}$, should be allocated appropriately to the selected links. Variable $u^{l}$ shows the number of teams assigned to link $l \in L^{(t)}$. We assume that the teams will not relocate among the selected links during their recovery processes and the next recovery decisions will be made when all the selected links are recovered. These assumptions are widely used in the literature. For example, Celik (2016) divide the planning horizon into equal time periods and recovery decisions are made at the beginning of each period. Averbakh (2012) also defines and applies a specific unit time for the restoration speed of the resources in a destroyed network. Tzeng et al. (2007) also define discrete time slots for the distribution of the relief supplies in post-disaster circumstances. Under these assumptions, we can show that:

Proposition 4: Sequential recovery of $n$ roads makes higher acceleration in the relief distribution than their simultaneous recovery. 
Therefore, one link should be selected from the set $L^{(t)}$ for recovery at each decision-making moment and all recovery teams should be assigned to that link. Selection of link $l$ from set $L^{(t)}$ for recovery depends on two factors: 1) How much $\theta^{p *}(\forall p \in P)$ values are affected by the recovery of link $l$ ? and 2) How long does it take to recover link l? The latter one depends on $\Lambda^{(t)}$. Set $E^{(t)}$ includes the links of $L^{(t)}$ that can be selected for recovery at time $t$ and their recovery can affect at least one of $\theta^{p *}(\forall p \in P)$ values. For each $l \in E^{(t)}$, the total improvement that the recovery of this link can make in the response cost of relief distribution would be as follows:

Proposition 5: If the link of $l \in E^{(t)}$ is selected for recovery at time $t$, and $\theta^{p *}$ values would improve to $\hat{\theta}^{p *}(l)$ after the recovery of this link, the total reduction in the response cost would be:

$\sum_{p \in P} \sum_{i=\eta^{p, t^{\prime}}(l)+1}^{\left[\frac{\Theta^{p}}{\vartheta^{p}}\right]} \vartheta^{p} \cdot c^{p}\left(\theta^{p *}+2 \theta^{p *}(i-1)\right)-\sum_{p \in P} \sum_{i=\eta^{p, t^{\prime}}(l)+1}^{\left[\frac{\Theta^{p}}{\vartheta^{p}}\right]} \vartheta^{p} \cdot c^{p}\left(\theta^{p *}+\right.$ $\left.2 \theta^{p *} \cdot\left(\eta^{p, t^{\prime}}(l)-1\right)+2 \dot{\theta}^{p *}(l) \cdot\left(i-\eta^{p, t^{\prime}}(l)\right)\right)$

where $\mathrm{t}^{\prime}(\mathrm{l})=\mathrm{t}+\frac{\mathrm{r}^{\mathrm{l}}}{\Lambda^{(\mathrm{t})}}$ and $\eta^{\mathrm{p}, \mathrm{t}^{\prime}}(\mathrm{l})=\left\lceil\frac{\mathrm{t}^{\prime}(\mathrm{l})+\theta^{\mathrm{p} *}}{2 \theta^{\mathrm{p} *}}\right\rceil$ show the recovery completion time for link $\mathrm{l}$ and the number of deliveries accomplished for pair $\mathrm{p} \in \mathrm{P}$ before the recovery completion time.

Following Averbakh (2012) and Celik et al. (2016), it is assumed that the transportation time of teams from their current location to the selected disrupted link is ignorable in comparison to its recovery time. To optimize the recovery decision made at time $t$, we select the best link from $E^{(t)}$ in a way to maximize the total reduction in the response cost: 


$$
\begin{gathered}
\underset{\forall l \in E^{(t)}}{\operatorname{MAX}}\left(\sum_{p \in P} \sum_{i=\eta^{p, t^{\prime}}(l)+1}^{\left\lceil\frac{\Theta^{p}}{\vartheta^{p}}\right]} \vartheta^{p} \cdot c^{p}\left(\theta^{p *}+2 \theta^{p *}(i-1)\right)-\sum_{p \in P} \sum_{i=\eta^{p, t^{\prime}}(l)+1}^{\left\lceil\frac{\Theta^{p}}{\vartheta^{p}}\right]} \vartheta^{p} \cdot c^{p}\left(\theta^{p *}+\right.\right. \\
\left.\left.2 \theta^{p *} \cdot\left(\eta^{p, t^{\prime}}(l)-1\right)+2 \dot{\theta}^{p *}(l) \cdot\left(i-\eta^{p, t^{\prime}}(l)\right)\right)\right)
\end{gathered}
$$

At time $t^{\prime}\left(l^{*}\right)$, the recovery process for the selected link will end and new transportation paths will be used for relief deliveries. Then, set $L^{(t)}$ will be updated by the new data collected by the UAVs (called $L^{\left(t^{\prime}\right)}$ ) and a new recovery decision (selecting a new link from the set $\mathrm{L}^{\left(\mathrm{t}^{\prime}\right)}$ and assigning recovery teams to that link) will be made similarly. This procedure will continue up to the moment that there are no unrecovered links in the network. 


\section{Chapter 4}

\section{COMPUTATIONAL RESULT}

The goals of this section are threefold: (i) we analyze and quantify the impact of the decisions made in Model 1 (prepositioning damage assessment facilities) on the performance of Model 2 (road recovery); (ii) we analyze and quantify the impact of considering Model 3 (relief distribution) in the decision making the process of Model 2 (road recovery); (iii) we compare the performance of our integrative method with an intuition-oriented benchmark that runs faster and finds good quality solutions. Current road recovery operations that are used in practice assume a pre-determined road prioritization. In practice, there is no systematic approach to prioritize roads (Celik et al. 2016). The benchmark heuristic is designed in a way to mimic current practice.

\subsection{Case Problem: Hurricane Scenarios}

We use the same case problem that was introduced in previous chapter. We define some scenarios representing the possible impacts of the hurricane on the target circle. On average, ten tropical storms are developed in the Atlantic Ocean, Gulf of Mexico and the Caribbean Sea per year. According to a 3-year average, six of the storms become hurricanes and five of them strike the US coastline from Texas to Maine. Two of these hurricanes are major. The size, intensity, speed, and direction of hurricanes vary considerably. The center of a hurricane is called the eye and the dense wall of thunderstorms surrounding the eye, approximately $20-25$ miles across, is called the eyewall. The eyewalls have the strongest and most destructive winds within hurricanes (NHC 2012). 
In Figure 9a, solid red lines show the track of the hurricane's cone that is predicted by the NHC. This cone includes our investigated area. The accuracy of predicting the cone track has improved a lot over time and its error has been reduced to 5.3 miles (NHC 2018). The dashed red lines show the track of the cone including its prediction errors on both sides. Red circles in Figure 9a represent the 5 scenarios defined for the movement track of the hurricane's eye and its surrounding eyewalls (with a diameter of 25 miles). We consider five levels of severity for the hurricane: In severity levels 1, 2, 3, 4, and 5, the number of randomly stricken nodes within the circles is $10-15,15-20,20-30,30-40$ and $40-50$, respectively (three repetitions). For the severity levels, the number of randomly disrupted roads is $20-30,30-40,40-50,50-60$ and 60-70 (two repetitions).
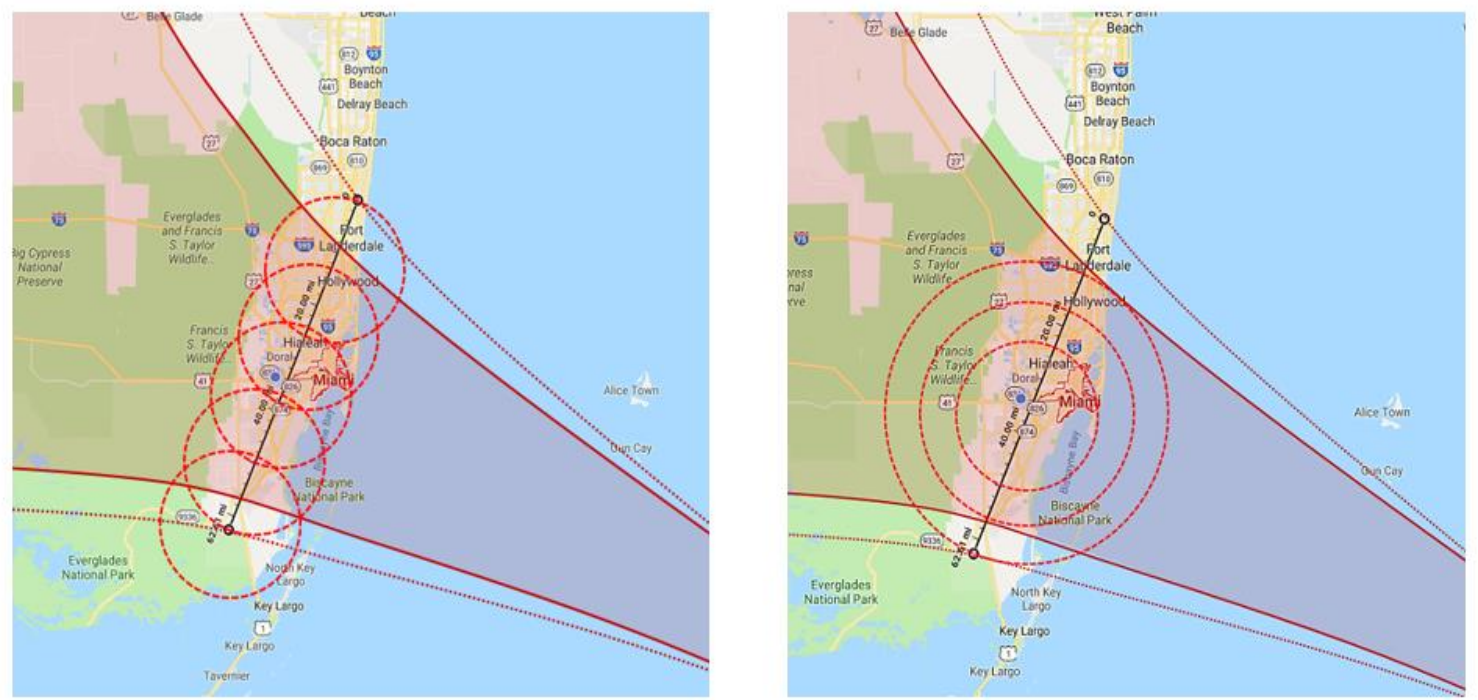

Figure 9. The zones of disrupted nodes and roads in the case problem. (a) The movement tracks for the eye. (b) The zones of block levels for roads.

The stricken nodes constitute the supply nodes in the network. As demand nodes, we consider two major hospitals in the area: Jackson Memorial Hospital and Memorial Regional Hospitals. These hospitals with respectively 1550 and 1022 beds are the biggest 
in the area. Following Celik et al. (2016), the supply quantity of each suburban region is estimated proportional to its population. Then, the aggregate supply is distributed to the nodes in proportion to the total length of the roads originating from the nodes. As shown in Figure 9b, the probability of disruption for the roads located in a distance of 12.5 (mile) from the eye is $60 \%$. For roads in distance 12.5-20.5 (mile) and >20.5 (mile), the disruption probability is $30 \%$ and $10 \%$, respectively. We also consider 3 debris levels for roads. In low, medium and high debris levels, the total time needed to traverse and recover a road is 60, 120, and 180 times of its travel time (that depends on its length) in unblocked conditions. In severity levels 1, 2, 3, 4 and 5, the blocked roads with debris levels of low, medium and high constitute $(80 \%, 15 \%$ and $5 \%),(70 \%, 20 \%$ and $10 \%),(50 \%, 30 \%$ and $20 \%),(20 \%, 30 \%$ and $50 \%)$ and $(10 \%, 20 \%$ and $70 \%)$ of all blocked roads. Each problem instance is solved for three different numbers of recovery teams $(3,5$, and 7 recovery teams) and three different scan completion times for the road network $(1.5,2.5$ and 3.5 days). The scan completion time depends on the number of UAVs located in Model 1. In total, 1350 problem instances are solved.

\subsection{Interaction between Model 1 and Model 2}

To analyze the interaction between Model 1 and Model 2, all of the problem instances generated are solved for three scan completion times: 1.5, 2.5 and 3.5 days. In the first case, enough UAVs are located in Model 1 to scan the whole network and provide the debris data of all blocked roads in 1.5 days after the landfall. In the second case, there are fewer located UAVs, and scan completion time is 2.5 days. The number of UAVs in the third case is less than the second one. So, the completion time increases to 3.5 days. 
The results are summarized in Table 2. Each cell of this table shows the average response cost for the 30 problem instances generated for the scan completion time, the number of recovery teams, and the disaster severity that correspond to that cell ( 5 scenarios for the eye's movement track $\times 3$ repetitions for stricken nodes selection $\times 2$ repetitions for blocked roads selection).

Table 2. The results for the problem instances.

\begin{tabular}{|c|c|c|c|c|c|c|c|c|c|}
\hline \multirow[b]{2}{*}{$\begin{array}{l}\text { Disaster } \\
\text { severity }\end{array}$} & \multicolumn{3}{|c|}{ Scan completion $=1.5$ days } & \multicolumn{3}{|c|}{ Scan completion $=2.5$ days } & \multicolumn{3}{|c|}{ Scan completion $=3.5$ days } \\
\hline & $\begin{array}{c}3 \\
\text { recovery } \\
\text { teams }\end{array}$ & $\begin{array}{c}5 \\
\text { recovery } \\
\text { teams }\end{array}$ & $\begin{array}{c}7 \\
\text { recovery } \\
\text { teams }\end{array}$ & $\begin{array}{c}3 \\
\text { recovery } \\
\text { teams }\end{array}$ & $\begin{array}{c}5 \\
\text { recovery } \\
\text { teams }\end{array}$ & $\begin{array}{c}7 \\
\text { recovery } \\
\text { teams }\end{array}$ & $\begin{array}{c}3 \\
\text { recovery } \\
\text { teams }\end{array}$ & $\begin{array}{c}5 \\
\text { recovery } \\
\text { teams }\end{array}$ & $\begin{array}{c}7 \\
\text { recovery } \\
\text { teams }\end{array}$ \\
\hline * & 608584 & 597004 & 595432 & 609004 & 599880 & 599256 & 619728 & 614848 & 613084 \\
\hline$* *$ & 897262 & 887198 & 877386 & 897578 & 888578 & 882890 & 900870 & 891298 & 890674 \\
\hline$* * *$ & 1334441 & 1304515 & 1296979 & 1337965 & 1318605 & 1312769 & 1340029 & 1321339 & 1319467 \\
\hline$* * * *$ & 1417870 & 1394304 & 1385976 & 1422750 & 1403342 & 1392346 & 1436110 & 1411850 & 1408018 \\
\hline$* * * * *$ & 1909112 & 1884056 & 1847876 & 1921324 & 1895544 & 1884124 & 1935904 & 1898468 & 1889688 \\
\hline $\begin{array}{ll}* & \text { Ins } \\
* * * & \text { Ins } \\
* * * * * & \text { Ins }\end{array}$ & inces with & severity le & $\begin{array}{l}\text { el } 1 \\
\text { el } 3 \\
\text { el } 5\end{array}$ & & & Instal & $\begin{array}{l}\text { es with } \\
\text { es with }\end{array}$ & $\begin{array}{l}\text { verity leve } \\
\text { verity leve }\end{array}$ & \\
\hline
\end{tabular}

As expected, the total response cost is an increasing function of scan completion time. Figure 10a shows the increment rate in the average response cost with respect to the scan completion time for different numbers of recovery teams. As seen in Figure 10a, increasing the scan completion time augments the increment rate of the response cost almost linearly for any number of recovery teams. On average, a unit increase in the completion time leads to 0.7 percent augmentation in the response cost. However, the increase in the increment rate is significantly higher when there are more recovery teams. Figure $10 \mathrm{~b}$ shows how the increment rate for a unit increase in the completion time augments with respect to the number of recovery teams. 

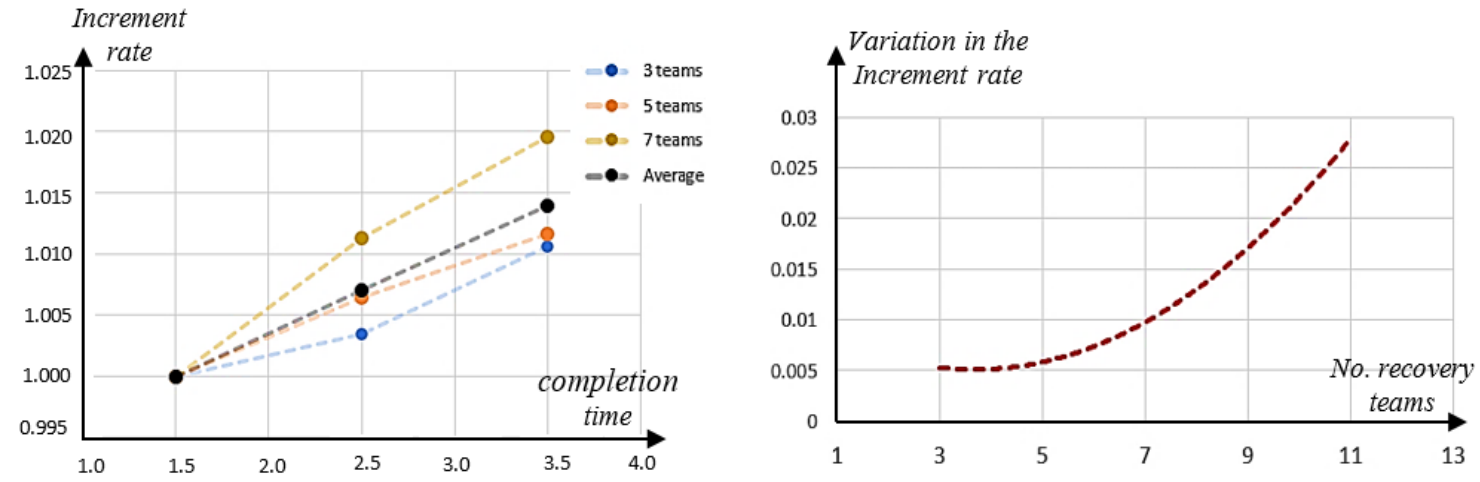

Figure 10. Variations in the total response cost. (a) Increment rate of the cost with respect to the scan completion time. (b) Increment rate of the cost with respect to number of recovery teams.

This result shows that fast data diffusion (in Model 1) results in a better recovery operation (in Model 2) when there are a higher number of recovery teams. When more recovery teams are available for restoration, the interaction between Model 1 and Model 2 is more significant.

Figure 11a shows the tradeoff between the pre-disaster preparedness cost (cost of Model 1) and post-disaster response cost (cost of Models 2 and 3). Prepositioning more UAVs in the preparedness phase increases the pre-disaster preparedness cost and also speeds up the data diffusion after the disaster. High data diffusion after the disaster augments the efficiency of road recovery and relief distribution operations. This leads to lower post-disaster response costs. This tradeoff demonstrates that the total cost is a convex function of scan completion time (see Figure 11b). The total cost has a minimum value at the intersection of these two functions, representing the pre-disaster preparedness cost and the post-disaster response cost. This is the best scan completion time minimizes the total cost by balancing the costs of preparedness and response operations. 

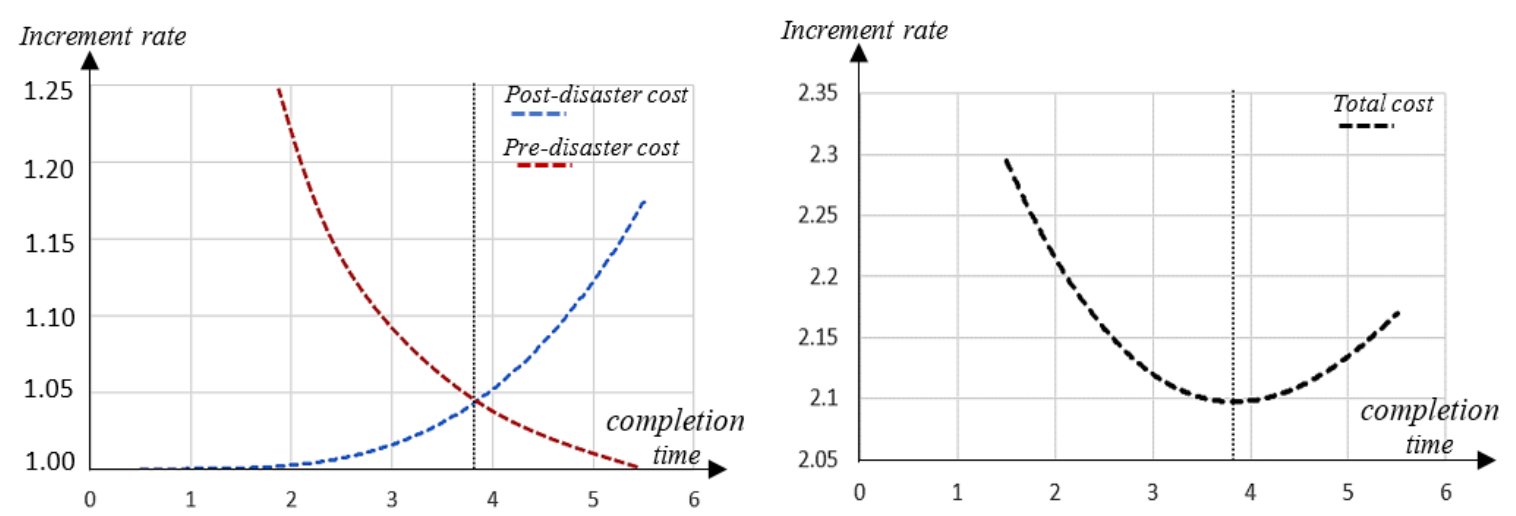

Figure 11. Increment rates of costs with respect to the scan completion time. (a) The increment rates of preand post-disaster costs with respect to the scan completion time. (b) The increment rate of the total cost with respect to the scan completion time.

\subsection{Interaction between Model 2 and Model 3}

In our approach, roads are selected for recovery (in Model 2) based on the impact they make in the relief distribution (Model 3). To highlight the importance of this integration, we change the selection criterion to another one that has been widely used in the literature. This new criterion is making reconnection in the network (among nodes or components) in the least possible time (Kasaei \& Salman 2016; Akbari \& Salman 2017; Celik et al. 2016). In this approach, at each decision-making time, road selection is done in a way to make the highest number of reconnections between the nodes in the network up to the next decision-making moment. Then the total response cost is calculated for this non-integrative approach and compared with the integrative approach proposed in previous chapter. The results are summarized in Table 3 and visualized in Figure 12. 


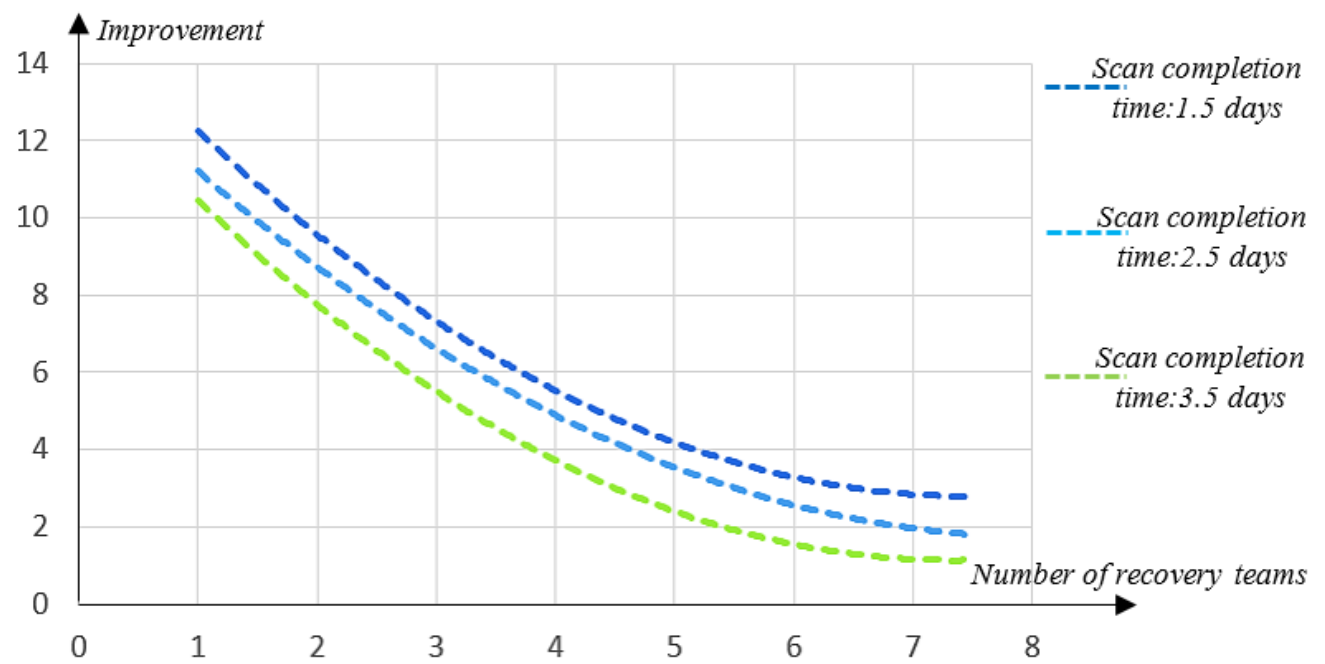

Figure 12. The improvement in the response cost.

As seen in Figure 12, the integration between Models 2 and 3 results in a higher reduction in the response cost when there is a smaller number of recovery teams and the damage/debris data is acquired quickly. While the scarcity of the recovery resources amplifies the importance of this integration, the benefit of the integration is mitigated by slow data diffusion. The improvement that is made by this integration increasingly boosts by the reduction in the number of recovery teams. In reducing the number of recovery teams from 7 to 5 and from 5 to 3 , the average increase in the improvement (cost reduction ratio) that is caused by a team reduction is $35 \%$ and $46 \%$, respectively. The improvement that is made by this integration decreasingly boosts by the reduction in the scan completion time (or the increment in the data diffusion speed). The average reduction in the response cost by reducing the scan completion time from 3.5 to 2.5 and from 2.5 to 1.5 is $34 \%$ and $18 \%$ respectively for any number of recovery teams. The number of recovery teams does not influence the impact of data diffusion speed on the response cost and vice versa. 
Table 3. The results for integrative and non-integrative approaches.

\begin{tabular}{|c|c|c|c|c|c|c|c|c|c|c|c|}
\hline \multirow{2}{*}{$\begin{array}{c}\text { Disaster } \\
\text { severity }\end{array}$} & $\begin{array}{c}\text { Solution } \\
\text { approach }\end{array}$ & 3 Scan completion $=1.5$ days & \multicolumn{2}{|c|}{ Scan completion $=2.5$ days } & \multicolumn{3}{|c|}{ Scan completion = 3.5 days } \\
\hline$*$ & 5 teams & 7 teams & 3 teams & 5 teams & 7 teams & 3 teams & 5 teams & 7 teams \\
\hline$*$ & IN & 608584 & 597004 & 595432 & 609004 & 599880 & 599256 & 619728 & 614848 & 613084 \\
\hline Improvement & $6.1 \%$ & $2.6 \%$ & $1.9 \%$ & $5.0 \%$ & $2.0 \%$ & $1.5 \%$ & $3.2 \%$ & $1.5 \%$ & $0.2 \%$ \\
\hline$* *$ & IN & 897262 & 887198 & 877386 & 897578 & 888578 & 882890 & 900870 & 891298 & 890674 \\
\hline$* *$ & non-IN & 956158 & 915446 & 900682 & 947762 & 906702 & 895726 & 933566 & 909606 & 899638 \\
\hline Improvement & $6.5 \%$ & $3.1 \%$ & $2.6 \%$ & $5.5 \%$ & $2.0 \%$ & $1.4 \%$ & $3.6 \%$ & $2.0 \%$ & $1.0 \%$ \\
\hline$* * *$ & IN & 1334441 & 1304515 & 1296979 & 1337965 & 1318605 & 1312769 & 1340029 & 1321339 & 1319467 \\
\hline$* * *$ & non-IN & 1420785 & 1360025 & 1333171 & 1415425 & 1366105 & 1332313 & 1404467 & 1335015 & 1339447 \\
\hline Improvement & $6.4 \%$ & $4.2 \%$ & $2.7 \%$ & $5.7 \%$ & $3.6 \%$ & $1.4 \%$ & $4.8 \%$ & $1.0 \%$ & $1.5 \%$ \\
\hline$* * * *$ & IN & 1417870 & 1394304 & 1385976 & 1422750 & 1403342 & 1392346 & 1436110 & 1411850 & 1408018 \\
\hline$* * * *$ & non-IN & 1539352 & 1473160 & 1436676 & 1541596 & 1472808 & 1431280 & 1541656 & 1452484 & 1427434 \\
\hline Improvement & $8.5 \%$ & $5.6 \%$ & $3.6 \%$ & $8.3 \%$ & $4.9 \%$ & $2.7 \%$ & $7.3 \%$ & $2.8 \%$ & $1.3 \%$ \\
\hline$* * * * *$ & IN & 1909112 & 1884056 & 1847876 & 1921324 & 1895544 & 1884124 & 1935904 & 1898468 & 1889688 \\
\hline$* * * * *$ & non-IN & 2084498 & 1987446 & 1939900 & 2087938 & 1994762 & 1937480 & 2102680 & 1989242 & 1923928 \\
\hline
\end{tabular}

\subsection{Benchmark approach}

To evaluate if and when the proposed approach is beneficial, we compare it with a simpler online benchmark approach. This benchmark mimics the current approach used by FEMA (FEMA 2018). The FEMA guideline suggests prioritizing the recovery of roads of higher convenience with regard to disaster response (roads for health centers and hospitals, main highways, etc.). Roads with less importance are recovered later. Mimicking the FEMA suggestions, we develop a benchmark approach that determines the recovery sequence at each decision-making moment according to three factors: Distance, Supply, and Debris. The distance factor represents the closeness of the road to the supply nodes. Since there is usually more than one supply node, this factor is calculated based on the 
distance between the road and the closest supply node to that road. The supply factor shows the expected supply from the closest supply node. The debris shows the road's debris level data provided by the UAVs. At each decision-making moment, the benchmark approach determines the recovery schedule for roads with known debris data according to $\frac{\text { supply }}{\text { distance } \times \text { debris }}$ ratio. The roads with higher ratios are scheduled to be recovered first. Figure 13 shows the differences between the post-disaster response costs of the integrative and benchmark approaches for different numbers of recovery teams and scan completion times. Table 4 summarizes the results obtained using the integrative and benchmark approaches for all the problem instances. The total improvement of the integrative approach over the benchmark is calculated as $\underline{\text { (Response cost of the benchmark-Response cost of the integrative approach) }}$

Response cost of the integrative approach

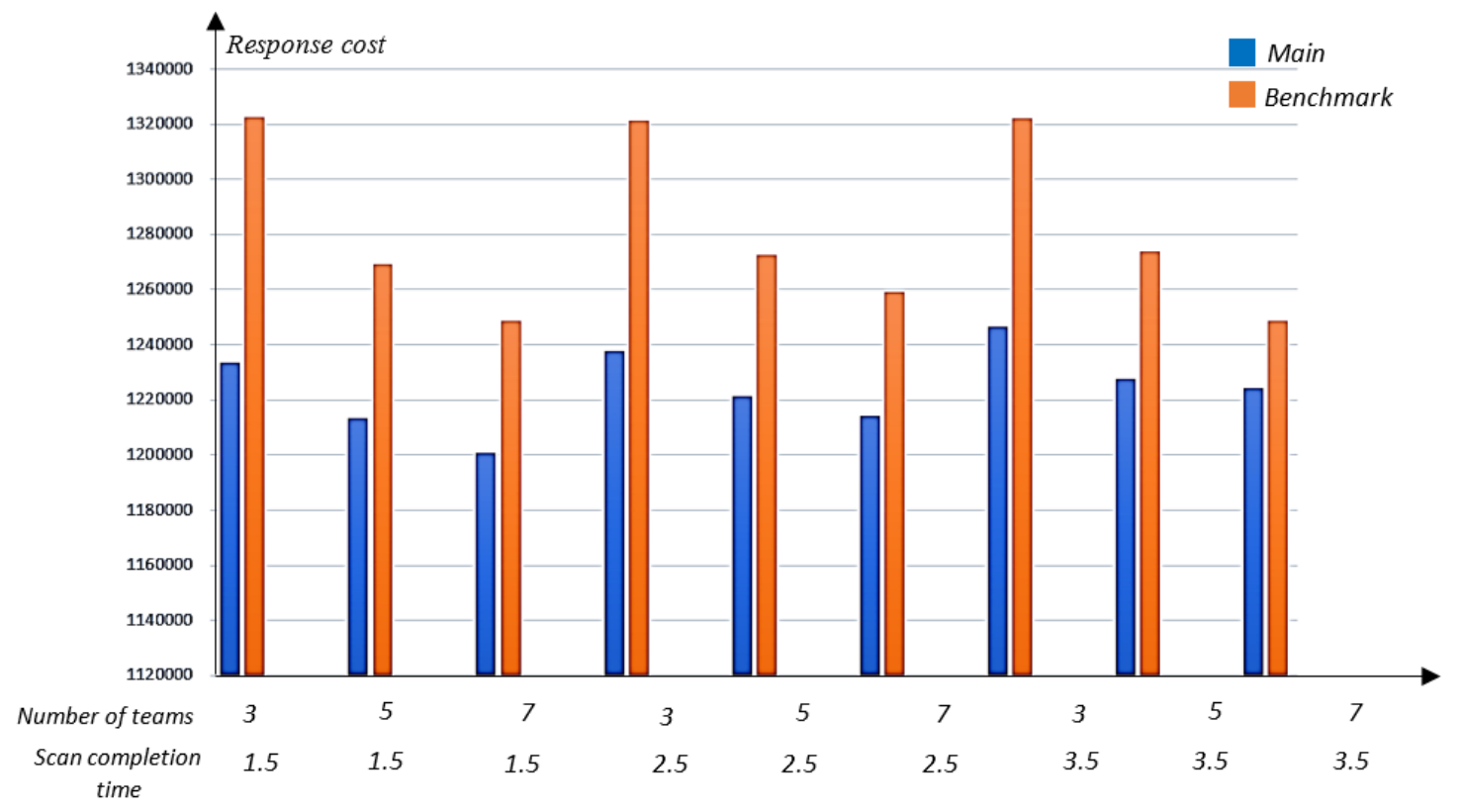

Figure 13. The differences between the post-disaster response costs. 
Table 4. The results for the integrative and benchmark approaches.

\begin{tabular}{|c|c|c|c|c|c|c|c|c|c|c|}
\hline \multirow{2}{*}{$\begin{array}{l}\text { Disaster } \\
\text { severity }\end{array}$} & \multirow{2}{*}{$\begin{array}{l}\text { Solution } \\
\text { approach }\end{array}$} & \multicolumn{3}{|c|}{$\begin{array}{c}\text { Scan completion }=1.5 \\
\text { days }\end{array}$} & \multicolumn{3}{|c|}{$\begin{array}{c}\text { Scan completion }=2.5 \\
\text { days }\end{array}$} & \multicolumn{3}{|c|}{$\begin{array}{c}\text { Scan completion }=3.5 \\
\text { days }\end{array}$} \\
\hline & & $\begin{array}{c}3 \\
\text { teams }\end{array}$ & $\begin{array}{c}5 \\
\text { teams }\end{array}$ & $\begin{array}{c}7 \\
\text { teams }\end{array}$ & $\begin{array}{c}3 \\
\text { teams }\end{array}$ & $\begin{array}{c}5 \\
\text { teams }\end{array}$ & $\begin{array}{c}7 \\
\text { teams }\end{array}$ & $\begin{array}{c}3 \\
\text { teams }\end{array}$ & $\begin{array}{c}5 \\
\text { teams }\end{array}$ & $\begin{array}{c}7 \\
\text { teams }\end{array}$ \\
\hline \multirow{3}{*}{$*$} & I & 608584 & 597004 & 595432 & 609004 & 599880 & 599256 & 619728 & 614848 & 613084 \\
\hline & B & 652456 & 626352 & 614888 & 655876 & 626484 & 608468 & 652692 & 629336 & 615732 \\
\hline & $\begin{array}{c}\text { improveme } \\
n t\end{array}$ & $7.2 \%$ & $4.9 \%$ & $3.3 \%$ & $7.7 \%$ & $4.4 \%$ & $1.5 \%$ & $5.3 \%$ & $2.3 \%$ & $0.43 \%$ \\
\hline \multirow{3}{*}{$* *$} & I & 897262 & 887198 & 877386 & 897578 & 888578 & 882890 & 900870 & 891298 & 890674 \\
\hline & B & 988706 & 925238 & 909586 & 956306 & 927554 & 907638 & 956306 & 922718 & 901558 \\
\hline & $\begin{array}{c}\text { improveme } \\
n t\end{array}$ & $10.2 \%$ & $4.3 \%$ & $3.7 \%$ & $6.5 \%$ & $4.4 \%$ & $2.8 \%$ & $6.1 \%$ & $3.5 \%$ & $1.2 \%$ \\
\hline \multirow{3}{*}{$* * *$} & I & 1334441 & 1304515 & 1296979 & 1337965 & 1318605 & 1312769 & 1340029 & 1321339 & 1319467 \\
\hline & B & 1411749 & 1360961 & 1344297 & 1419093 & 1365313 & 1342281 & 1420765 & 1363201 & 1342685 \\
\hline & $\begin{array}{c}\text { improveme } \\
n t\end{array}$ & $5.8 \%$ & $4.3 \%$ & $3.6 \%$ & $6.1 \%$ & $3.5 \%$ & $2.2 \%$ & $6.1 \%$ & $3.2 \%$ & $1.8 \%$ \\
\hline \multirow{3}{*}{$* * * *$} & I & 1417870 & 1394304 & 1385976 & 1422750 & 1403342 & 1392346 & 1436110 & 1411850 & 1408018 \\
\hline & B & 1517674 & 1465758 & 1438052 & 1520638 & 1468178 & 1441256 & 1524294 & 1467530 & 1436246 \\
\hline & $\begin{array}{c}\text { improveme } \\
n t\end{array}$ & $7 \%$ & $5.1 \%$ & $3.8 \%$ & $6.9 \%$ & $4.6 \%$ & $3.5 \%$ & $6.1 \%$ & $3.9 \%$ & $2 \%$ \\
\hline \multirow{3}{*}{$* * * * *$} & I & 1909112 & 1884056 & 1847876 & 1921324 & 1895544 & 1884124 & 1935904 & 1898468 & 1889688 \\
\hline & $B$ & 2043156 & 1967122 & 1936356 & 2054240 & 1975290 & 1945432 & 2056472 & 1987306 & 1946920 \\
\hline & $\begin{array}{c}\text { improveme } \\
n t\end{array}$ & $7 \%$ & $4.4 \%$ & $4.8 \%$ & $6.9 \%$ & $4.2 \%$ & $3.2 \%$ & $6.2 \%$ & $4.7 \%$ & $3 \%$ \\
\hline
\end{tabular}

The improvement trends in the results demonstrate in what situations the proposed approach is more effective. We investigate the trends with respect to the number of recovery teams and the scan completion time. Figure 14a shows the trend of improvements with respect to the number of recovery teams. The improvement made by the integrative approach is higher when there is a smaller number of recovery teams. The average improvement for the problem instances with 3,5 and 7 recovery teams is $6.75 \%, 4.13 \%$, and $2.97 \%$, respectively. This result shows that the efficiency of the integrative approach is more significant when there is a recovery resource scarcity. Increasing the recovery 
resource reduces the efficiency of the integrative approach in comparison with the benchmark approach, mimicking the FEMA approach. This reduction trend is concave-up; the reduction for a unit increase in the number of the recovery teams is high when there is a small number of recovery teams and becomes smaller by increasing the number of recovery teams.

Figure $14 \mathrm{~b}$ shows the sensitivity of the improvement with respect to the scan completion time determined in Model 1 (another interaction between Models 1 and 2). The average improvement made by the integrative approach is higher in short scan completion time. In the case problem, the average improvement for the problem instances with 1.5, 2.5 and 3.5 scan completion time is $5.30 \%, 4.82 \%$, and $3.73 \%$, respectively. The proposed approach makes more improvement when a higher number of roads is available for selection at each decision-making moment. This demonstrates the sensitivity of the integrative method with respect to the data diffusion speed. Fast data diffusion in postdisaster circumstances augments the efficiency of the proposed approach with respect to the benchmark mimicking the FEMA approach. The reduction trend is concave-down. The reduction for a unit increase in the scan completion time is low at small values of scan completion time and the reduction becomes higher by increasing the scan time. 

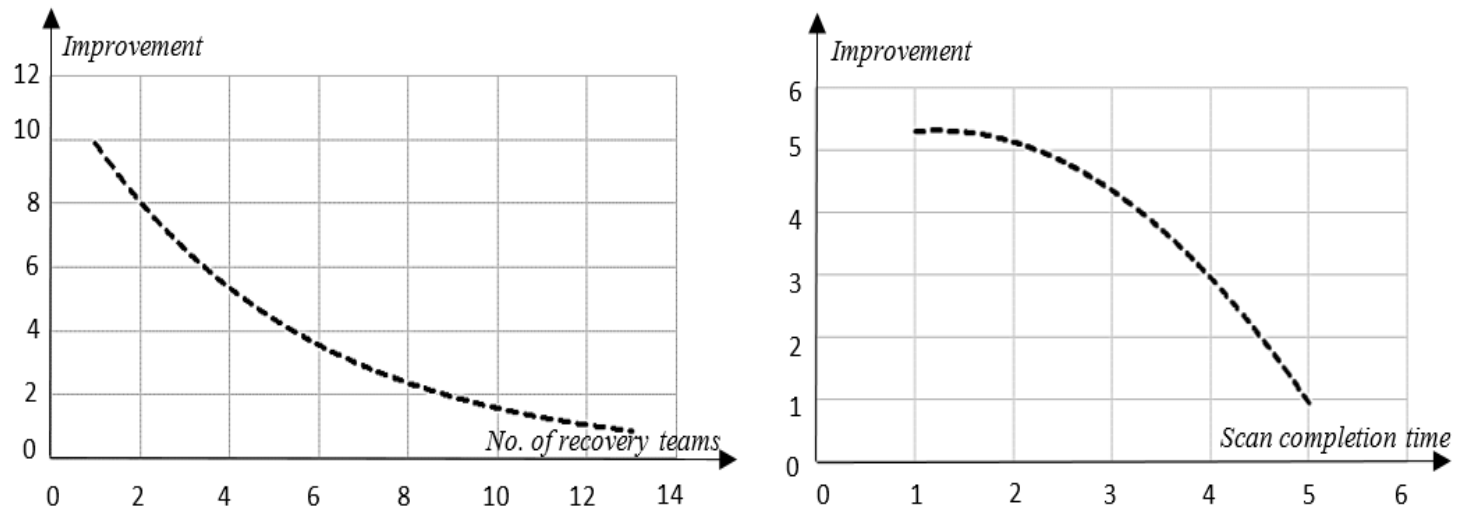

Figure 14. The improvement trends. (a) Improvement with respect to the number of recovery teams. (b) Improvement with respect to the scan completion time. 


\section{Chapter 5}

\section{CONCLUSION}

In this study, we develop an integrative online optimization model with a modelbased data diffusion component to coordinate three restoration-interdependent operations in the disaster response phase such as "damage assessment", "road recovery" and "relief distribution". An optimization model is developed for the damage assessment operation. This model prepositions UAVs and their charging stations over the network and determines their moving walks to scan the network and diffuse the damage/debris data. This data is

periodically transferred to the second model developed for road recovery (a directional interdependency between two operations). An online optimization model is developed for road recovery. This model prioritizes the recovery process of roads with known damage/debris data in a way to make the highest acceleration in relief distribution operation (a mutual interdependency between two operations). We use the integrative framework to determine the importance of interactions between these three operations. Results show that:

- Interaction between the damage assessment \& road recovery: Fast data diffusion in the damage assessment operation results in a better road recovery operation when there is a higher number of recovery teams. The scarcity of the recovery resources neutralizes the impact of fast data diffusion. There is an optimal data diffusion speed (or scan completion time) that balances the pre-disaster preparedness and the post-disaster response costs.

- Interaction between the road recovery \& relief distribution: The scarcity of the recovery resources amplifies the importance of integration between road recovery and 
relief distribution operations. However, the benefit of this integration is mitigated by slow data diffusion.

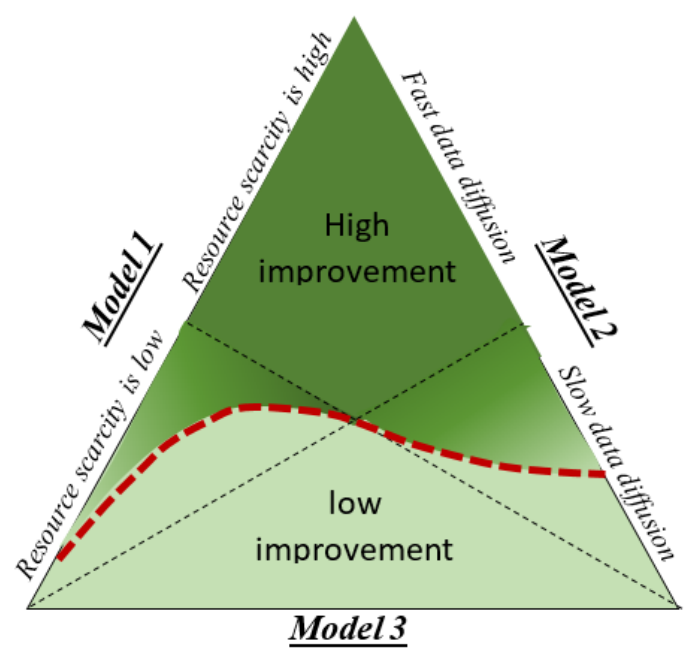

Figure 15. The conditions under which the integrative approach is more effective.

The performance of the proposed approach is compared with an online heuristic benchmark mimicking the performance of FEMA. The results show that the integrative approach makes more significant improvements when the recovery resource is scarce, and the data diffusion is fast. Increasing the recovery resource and slowing down the data diffusion speed makes concave-up and concave-down reductions in the improvement made by the integrative approach, respectively. Figure 15 demonstrates the conditions under which the proposed integrative approach is more effective.

In this study, we assume that damage assessment facilities, UAVs, are prepositioned before the disaster and their moving walks are not adjusted after the disaster. Post-disaster adjustments of the walks may lead to fast data diffusion and higher improvements and are suggested as a future research direction for the investigated problem of this study. 


\section{REFERENCES}

Agatz, N., Bouman, P., and Schmidt, M. (2018). Optimization approaches for the traveling salesman problem with drone. Transportation Science, 52(4): 965-981.

Akbari, V., and Salman, F. S. (2017). Multi-vehicle synchronized arc routing problem to restore post-disaster network connectivity. European Journal of Operational Research, 257(2): 625-640.

Aksu, D. T., and Ozdamar, L. (2014). A mathematical model for post-disaster road restoration: Enabling accessibility and evacuation. Transportation Research Part E: Logistics and Transportation Review, 61: 56-67.

Altay, N., and Green III, W. G. (2006). OR/MS research in disaster operations management. European journal of operational research, 175(1): 475-493.

Amazon (2016). Amazon prime air. https://www.amazon.com/b?node=8037720011. (Accessed on 11/19/2017).

Apte, A. (2010). Humanitarian logistics: A new field of research and action. Foundations and trends $®$ in technology, information and operations management, 3(1): 1-100.

Averbakh, I. (2012). Emergency path restoration problems. Discrete Optimization, 9(1), 58-64.

Balcik, B. (2017). Site selection and vehicle routing for post-disaster rapid needs assessment. Transportation research part E: logistics and transportation review, 101: 3058.

Bertsimas, D., Brown, D. B., and Caramanis, C. (2011). Theory and applications of robust optimization. SIAM review, 53(3): 464-501.

Camacho-Vallejo, J. F., González-Rodríguez, E., Almaguer, F. J., and GonzálezRamírez, R. G. (2015). A bi-level optimization model for aid distribution after the occurrence of a disaster. Journal of Cleaner Production, 105: 134-145.

Campbell, A. M., Vandenbussche, D., and Hermann, W. (2008). Routing for relief efforts. Transportation Science, 42(2): 127-145.

Carlsson, J. G., and Song, S. (2018). Coordinated logistics with a truck and a drone. Management Science, 64(9): 4052-4069.

Çelik, M. (2016). Network restoration and recovery in humanitarian operations: Framework, literature review, and research directions. Surveys in Operations Research and Management Science, 21(2): 47-61. 
Çelik, M., Ergun, Ö., and Keskinocak, P. (2015). The post-disaster debris clearance problem under incomplete information. Operations Research, 63(1): 65-85.

Choi-Fitzpatrick, A., Chavarria, D., Cychosz, E., Dingens, J. P., Duffey, M., Koebel, K., Siriphanh, S., Yurika Tulen, M., Watanabe, H., Juskauskas, T., et al. (2016). Up in the Air: A Global Estimate of Non-Violent Drone Use 2009-2015.

Chowdhury, S., Emelogu, A., Marufuzzaman, M., Nurre, S. G., and Bian, L. (2017). Drones for disaster response and relief operations: A continuous approximation model. International Journal of Production Economics, 188: 167-184.

Coleman, D., Georgiadou, Y., and Labonte, J. (2009). Volunteered geographic information: The nature and motivation of produsers. International journal of spatial data infrastructures research, 4(4): 332-358.

DHL (2014) Dhl_-press release — english. http://www.dhl.com/en/press/releases/releases _ 2014/group/dhl _ parcelcopter_ launches _ initial _ operations _ for _ research _ purposes.html. (Accessed on 11/19/2017)

Dunke, F., and Nickel, S. (2016). A general modeling approach to online optimization with lookahead. Omega, 63: 134-153.

Farahani, R. Z., Asgari, N., Heidari, N., Hosseininia, M., and Goh, M. (2012). Covering problems in facility location: A review. Computers \& Industrial Engineering, 62(1): 368407.

Federal Emergency Management Agency (2018) National Disaster Recovery Framework https://www.fema.gov/national-disaster-recovery-framework. (Retrieved on April 3, 2019)

Fetter, G., and Falasca, M. (2011) Establishing the need for decision support in disaster debris disposal. In Proceedings of the 8th International ISCRAM Conference, (Lisbon, Portugal) (pp. 1-4).

Ghiani, G., Laporte, G., and Musmanno, R. (2004). Introduction to logistics systems planning and control. John Wiley and Sons.

Google (2014) Google tests drone deliveries in project wing trials - BBC news. http://www.bbc.com/news/technology-28964260. (Accessed on 11/19/2017).

Gupta, S., Starr, M. K., Farahani, R. Z., and Matinrad, N. (2016). Disaster management from a POM perspective: Mapping a new domain. Production and Operations Management, 25(10): 1611-1637. 
Huang, H., Long, J., Wu, Y., Yi, Q., Zhang, G., and Lei, B. (2017). A method for using unmanned aerial vehicles for emergency investigation of single geo-hazards and sample applications of this method. Natural Hazards and Earth System Sciences, 17(11): 1961.

Joint, F. A. O. (2017) Drone mapping technology for enhanced disaster risk reduction and management in the agriculture sector.

Kasaei, M., and Salman, F. S. (2016). Arc routing problems to restore connectivity of a road network. Transportation Research Part E: Logistics and Transportation Review, 95: 177-206.

Kibar AN, Salman S (2013) Logistics planning for restoration of network connectivity after a disaster. Working paper, Department of Industrial Engineering, Koç University, Ankara, Turkey.

Liberatore, F., Ortuño, M. T., Tirado, G., Vitoriano, B., and Scaparra, M. P. (2014). A hierarchical compromise model for the joint optimization of recovery operations and distribution of emergency goods in Humanitarian Logistics. Computers and Operations Research, 42: 3-13.

Lim, G. J., Kim, S., Cho, J., Gong, Y., and Khodaei, A. (2016). Multi-UAV prepositioning and routing for power network damage assessment. IEEE Transactions on Smart Grid, 9(4): 3643-3651.

Luis, E., Dolinskaya, I. S., and Smilowitz, K. R. (2012). Disaster relief routing: Integrating research and practice. Socio-economic planning sciences, 46(1): 88-97.

Luther, L. G., and Resources, Science, and Industry Division. (2006, June). Disaster debris removal after Hurricane Katrina: Status and associated issues. Congressional Research Service, the Library of Congress.

Hildmann, H., and Kovacs, E. (2019). Using Unmanned Aerial Vehicles (UAVs) as Mobile Sensing Platforms (MSPs) for Disaster Response, Civil Security and Public Safety. Drones, 3(3): 59.

Molina, P., Colomina, I., Victoria, T., Skaloud, J., Kornus, W., Prades, R., and Aguilera, C. (2012). Searching lost people with UAVs: the system and results of the close-search project. International Archives of the Photogrammetry, Remote Sensing and Spatial Information Sciences, 39(CONF): 441-446.

National Hurricane Centre (2012). Saffir-Simpson Hurricane Wind Scale, http://www.nhc.noaa.gov/aboutsshws.php, Retrieved on August 24, 2018.

National Hurricane Centre (2018). Definition of the NHC Track Forecast Cone, http://www.nhc.noaa.gov/aboutcone.shtml, Retrieved on August 24, 2018. 
Nedjati, A., Vizvari, B., and Izbirak, G. (2016). Post-earthquake response by small UAV helicopters. Natural Hazards, 80(3): 1669-1688.

Nurre, S. G., and Sharkey, T. C. (2014). Integrated network design and scheduling problems with parallel identical machines: Complexity results and dispatching rules. Networks, 63(4): 306-326.

Oruc, B. E., and Kara, B. Y. (2018). Post-disaster assessment routing problem. Transportation research part B: methodological, 116: 76-102.

Otto, A., Agatz, N., Campbell, J., Golden, B., and Pesch, E. (2018). Optimization approaches for civil applications of unmanned aerial vehicles (UAVs) or aerial drones: A survey. Networks, 72(4): 411-458.

Özdamar, L., Aksu, D. T., and Ergüneş, B. (2014). Coordinating debris cleanup operations in post disaster road networks. Socio-Economic Planning Sciences, 48(4): 249-262.

Özdamar, L., and Ertem, M. A. (2015). Models, solutions and enabling technologies in humanitarian logistics. European Journal of Operational Research, 244(1): 55-65.

Perrier, N., Langevin, A., and Amaya, C. A. (2008). Vehicle routing for urban snow plowing operations. Transportation Science, 42(1): 44-56.

POLICY O (2014) Unmanned Aerial Vehicles in Humanitarian. STUDIES, 10.

Sahin H, Kara BY, Karasan O (2013) On debris removal during the response phase. Working paper, Department of Industrial Engineering, Bilkent University, Ankara, Turkey.

Sahin, H., Kara, B. Y., and Karasan, O. E. (2016). Debris removal during disaster response: A case for Turkey. Socio-economic planning sciences, 53: 49-59.

Salmerón, J., and Apte, A. (2010). Stochastic optimization for natural disaster asset prepositioning. Production and operations management, 19(5): 561-574.

Scott, J., and Scott, C. (2017, January). Drone delivery models for healthcare. In Proceedings of the 50th Hawaii international conference on system sciences.

Tzeng, G. H., Cheng, H. J., and Huang, T. D. (2007). Multi-objective optimal planning for designing relief delivery systems. Transportation Research Part E: Logistics and Transportation Review, 43(6): 673-686. 
Vanajakumari, M., Kumar, S., and Gupta, S. (2016). An integrated logistic model for predictable disasters. Production and Operations Management, 25(5): 791-811.

Yan, S., and Shih, Y. L. (2009). Optimal scheduling of emergency roadway repair and subsequent relief distribution. Computers \& Operations Research, 36(6): 2049-2065.

Zheng, Y. J., Du, Y. C., Sheng, W. G., and Ling, H. F. (2019). Collaborative HumanUAV Search and Rescue for Missing Tourists in Nature Reserves. Interfaces, 49(5): 371 383. 


\section{APPENDIX A}

Table 1A includes the list of notations used in the paper.

Table 1A. Notation.

\begin{tabular}{|c|c|}
\hline Set & definition \\
\hline$N$ & Set of nodes in the original road network \\
\hline$L$ & Set of links in the original road network \\
\hline$\hat{N}$ & Set of nodes in the unified network (including the original and auxiliary nodes) \\
\hline $\begin{array}{l}L \\
\end{array}$ & Set of links in the unified network \\
\hline$T$ & Set of decision-making time steps for road selection \\
\hline$N^{\prime \prime}$ & Set of nodes in the super-network \\
\hline$L^{\prime \prime}$ & Set of links in the super-network \\
\hline$\hat{L}^{P}$ & Set of prioritized roads $\left(L^{P} \subset L\right)$ \\
\hline$\hat{L}^{U}$ & Set of non-prioritized roads $\left(\hat{L}^{U}=L_{L}-\hat{L}^{P}\right)$ \\
\hline$P$ & $\begin{array}{l}\text { The set of all pairs of demand and supply nodes that appear after the disaster } P= \\
\{p=(n, m) \mid n, m \in N\}\end{array}$ \\
\hline$L^{(t)}$ & The set of disrupted roads that are scanned by the UAVs up to time $t$ \\
\hline$E^{(t)}$ & $\begin{array}{l}\text { The subset of links of } L^{(t)} \text { that can be selected for recovery at time } t \text { and their } \\
\text { recovery can affect at least one of } \theta^{p *}(\forall p \in P) \text { values. }\end{array}$ \\
\hline Variable & definition \\
\hline$z_{n}$ & 1 if a charging station is located at node $n$; and 0 otherwise \\
\hline$x_{n}$ & The number of UAVs prepositioned at node $n$ \\
\hline$y_{(\overrightarrow{n, m})}^{t}$ & The number of UAVs flying through link $(\overrightarrow{n, m})$ at time step $t$ \\
\hline$\Psi$ & The scan completion time \\
\hline$\left.w_{(\overrightarrow{m n}}^{t}\right)$ & 1 if a UAV fly through link $(\overrightarrow{n, m})$ at time step $t$, and 0 otherwise \\
\hline$\eta$ & $\begin{array}{l}\text { The number of times that the links of the network are traversed more than once by } \\
\text { the UAVs }\end{array}$ \\
\hline$\Psi^{\operatorname{Min}}\left(n^{U}\right)$ & The minimum $\Psi$ to scan the whole network with $n^{U}$ number of UAVs \\
\hline$\Omega$ & The scan completion time for the prioritized roads \\
\hline$\dot{x}_{n}$ & The total amount of relief flow originating from node $n$ in each delivery \\
\hline$\left.\dot{y}_{(\overrightarrow{n, m} t}^{t}\right)$ & The total amount of relief flows through the link $(n, m)$ at time $t$; and 0 otherwise \\
\hline$w_{(\overrightarrow{n, m})}^{t}$ & 1 if the relief flows through the link $(n, m)$ at time $t$; and 0 otherwise \\
\hline$\theta^{p}$ & The shortest transportation time between the nodes of pair $p \in P$ \\
\hline$u^{l}$ & The number of teams assigned to link $l \in L^{(t)}$ \\
\hline$t^{\prime}(l)$ & The recovery completion time for link $l$ \\
\hline$\eta^{p, t^{\prime}}(l)$ & $\begin{array}{l}\text { The number of deliveries accomplished for pair } p \in P \text { before the recovery } \\
\text { completion time of link } l\end{array}$ \\
\hline$\dot{\theta}^{p *}(l)$ & $\begin{array}{l}\text { The shortest transportation time between the nodes of pair } p \in P \text { after recovering link } \\
l\end{array}$ \\
\hline Parameter & definition \\
\hline$v$ & The velocity of the UAVs \\
\hline$N D U$ & $\begin{array}{l}\text { The largest distance that the length of all roads and the fly distance of the UAVs can } \\
\text { be estimated as a coefficient of it }\end{array}$ \\
\hline
\end{tabular}




\begin{tabular}{|c|l|}
\hline$d$ & The highest number of NDU that a fully-charged UAV can fly \\
\hline$f_{n}$ & The cost of establishing a charging station at node $n$ \\
\hline$p_{n}$ & The cost of deploying a UAV at node $n$ \\
\hline$o$ & The operational cost of moving a UAV over a road per NDU \\
\hline$a_{(n, m)}$ & 1 if there is a link between nodes $n$ and $m ;$ and 0 otherwise \\
\hline$\dot{a}_{(n, m)}$ & The number of links between nodes $n$ and $m$ in the super-network \\
\hline $\bar{d}_{(n, m)}$ & The average distance between nodes $n$ and $m$ in the super-network \\
\hline$\beta_{(n, m)}$ & 1 if link $(n, m) \in \dot{L}^{P} ;$ and 0 otherwise \\
\hline$\zeta$ & This parameter represents the largest integer value that $\zeta d \leq|T|$ \\
\hline$n^{U}$ & A fixed number of UAVs \\
\hline$n^{S}$ & A fixed number of charging stations \\
\hline$M$ & A very large constant value \\
\hline$\dot{\psi}$ & A fixed predetermined value for variable $\Psi$ \\
\hline$\Theta^{p}$ & The total amount of shipment between the nodes of pair $p \in P$ \\
\hline$\vartheta^{p}$ & The capacity of the transportation fleet assigned to pair $p \in P$ \\
\hline$c^{p}(t)$ & The cost of fulfilling a unit of demand at the demand node of pair $p$ at time $t$ \\
\hline$\gamma_{(n, m)}$ & $\begin{array}{l}1 \text { if link }(n, m) \text { is undisrupted and can be used by the transportation fleet, and } 0 \\
\text { otherwise }\end{array}$ \\
\hline$\gamma_{n}^{p}$ & 1 for node $n \in N$ if it is the supply node of pair $p ;$ and 0 otherwise \\
\hline$\dot{\gamma}_{n}^{p}$ & 1 for node $n \in N$ if it is the supply node of pair $p ;$ and 0 otherwise \\
\hline$r^{l}$ & The required recovery time for link $l \in L^{(t)}$ if it is recovered by a single team \\
\hline$\Lambda^{(t)}$ & The number of recovery teams available at time $t$ \\
\hline
\end{tabular}

\section{APPENDIX B}

Proof of Proposition 1: If we assume that the scan completion time is $\Psi$, the maximum number of NDU that can be traversed by $\mathrm{n}^{\mathrm{U}}$ number of UAV is $\Psi n^{U}$. On the other side, the travel distance of UAVs should be more than or equal to $|\hat{L}|$ to ensure that all roads of the network are scanned. This necessitates that $\Psi n^{U}=|\dot{L}|+\eta . \eta$ is a non-negative integer value representing the number of times the links of the network, $G$, are traversed more than one time by the UAVs. For efficient UAV prepositioning, the model forces the extra distance traversed by UAVs, $\eta$, to zero. So, even in the optimal prepositioning, the scan completion time cannot be less than $\left\lceil\frac{|L|}{n^{U}}\right]$.

Proof of Proposition 2: The distance between two adjacent charging stations (two charging stations are called adjacent if there is no other charging station in their shortest path) cannot be more than d. Otherwise, a UAV leaving one of these stations will become out-of-charge before reaching the other one. When $\Psi$ is less than $d$, one UAV from one of the stations cannot scan the whole links 
between two adjacent stations within $\Psi$. So, two UAVs from the two stations will concurrently scan the links in opposite directions. Therefore, the distance between the two stations should be $2 \Psi$. Considering the two cases $(\Psi \geq d \& \Psi<d)$, the distance between two adjacent stations in a network with $n^{U}$ number of UAVs and scan completion time of $\Psi^{\operatorname{Min}}\left(n^{U}\right)$ should be $\operatorname{Min}\left\{2 \Psi^{\operatorname{Min}}\left(n^{U}\right), d\right\}$. There are $|\hat{L}|$ links and the highest number of stations is needed when all links are sequentially connected and form a loop which is equal to $\left\lceil\frac{|\dot{L}|}{\operatorname{Min}\left\{2 \Psi^{\operatorname{Min}}\left(n^{U}\right), d\right\}}\right\rceil$. So, in general networks $n^{S} \leq\left\lceil\frac{|\dot{L}|}{\operatorname{Min}\left\{2 \Psi^{\operatorname{Min}}\left(n^{U}\right), d\right\}}\right\rceil$. Finding the exact value for $n^{S}$ without a model is impossible because it completely depends on the topology of the network. Model P2 locates charging stations in a network in a way that the distance between any pair of stations is less than or equal to $\operatorname{Min}\left\{2 \Psi^{\operatorname{Min}}\left(n^{U}\right), d\right\}$ (Constraints 17-19). Constraints (20-21) ensures that all the links of the network should be covered by the paths considered between the stations.

Proof of Proposition 3: Since $\vartheta^{\mathrm{p}}$ units of the product can be shipped in each delivery, the total number of delivery frequency between supply and demand nodes of pair $p(\forall p \in P)$ would be $\left\lceil\frac{\Theta^{p}}{\vartheta^{p}}\right\rceil$. The first delivery is materialized at the time $\theta^{p *}$. Then, $2 \theta^{p *}$ time units are needed for the vehicle to return the supply node, load and move toward the demand node. So, $i^{\text {th }}$ delivery happens at the time $\theta^{p *}+2 \theta^{p *}(i-1)$. The response cost for the $i^{\text {th }}$ delivery of pair $p$ that includes $\vartheta^{p}$ product units would be $\vartheta^{p} \cdot c^{p}\left(\theta^{p *}+2 \theta^{p *}(i-1)\right)$. So, the total response cost for all demand and supply pairs in the network bould $\sum_{p \in P} \sum_{i=1}^{\left[\frac{\Theta^{p}}{\vartheta^{p}}\right]} \vartheta^{p} \cdot c^{p}\left(\theta^{p *}+2 \theta^{p *}(i-1)\right)$

Proof of Proposition 4: Assume that there are $n$ links with the recovery times of $r^{1}, r^{2}, \ldots, r^{n}$ and the total number of recovery teams that can be assigned to the links is $\Lambda^{(\mathrm{t})}$. If we decide to simultaneously recover all of these links, the recovery teams should be distributed among the links 
as $\mathrm{u}^{1}, \mathrm{u}^{2}, \ldots, \mathrm{u}^{\mathrm{n}}\left(\Lambda^{(\mathrm{t})}=\sum_{i=1}^{n} u^{i}\right.$ and $\left.\forall u^{i}>0\right)$. In this case, the recovery will end at $\operatorname{Max}\left\{\frac{r^{1}}{u^{1}}, \frac{r^{2}}{u^{2}}, \ldots, \frac{r^{n}}{u^{n}}\right\}$. However, if we decide to recover the links sequentially, the recovery will end at $\frac{r^{1}+r^{2}+\cdots+r^{n}}{u^{1}+u^{2}+\cdots+u^{n}}$. Following the fact that $\operatorname{Max}\left\{\frac{r^{1}}{u^{1}}, \frac{r^{2}}{u^{2}}, \ldots, \frac{r^{n}}{u^{n}}\right\} \geq \frac{r^{1}+r^{2}+\cdots+r^{n}}{u^{1}+u^{2}+\cdots+u^{n}}$, we can conclude that sequential recovery is always better than simultaneous recovery.

Proof of Proposition 5: $\mathrm{r}^{\mathrm{l}}$ shows the recovery time of link $\mathrm{l} \in \mathrm{L}^{(\mathrm{t})}$ if it is recovered by a single team. Since $\Lambda^{(\mathrm{t})}$ teams are available at time $t$, the recovery of link $l$ will last $\frac{r^{l}}{\Lambda^{(t)}}$. This means that if we select link $l$ for recovery at time $t$, the recovery process of this link will end at the time $t^{\prime}(l)=t+$ $\frac{r^{l}}{\Lambda^{(t)}}$. So, the number of deliveries that their transportation will start before retrieving road $l$ is $\eta^{p, t^{\prime}}(l) . \quad \eta^{p, t^{\prime}}(l)$ is the lowest integer value that satisfies the following inequality: $\theta^{p *}+$ $2 \theta^{p *}\left(\eta^{p, t^{\prime}}(l)-1\right) \geq t^{\prime}(l)$. Simplifying this inequality shows that $\eta^{p, t^{\prime}}(l)=\left\lceil\frac{t^{\prime}(l)+\theta^{p *}}{2 \theta^{p *}}\right\rceil$. If link $l$ will not be recovered, the response cost of $\eta^{p, t^{\prime}}(l)+1^{\text {th }}$ to $\left\lceil\frac{\Theta^{p}}{\vartheta^{p}}\right\rceil^{\text {th }}$ deliveries would be $\sum_{p \in P} \sum_{i=\eta^{p, t^{\prime}}(l)+1}^{\left\lceil\frac{\Theta^{p}}{\vartheta^{p}}\right\rceil} \vartheta^{p} \cdot c^{p}\left(\theta^{p *}+2 \theta^{p *}(i-1)\right)$. If link $l$ will be recovered, the transportation time for $\eta^{p, t^{\prime}}(l)+1^{\text {th }}$ to $\left\lceil\frac{\Theta^{p}}{\vartheta^{p}}\right\rceil^{\text {th }}$ deliveries will decrease and the delivery time for the $i^{\text {th }}$ delivery $\left(\eta^{p, t^{\prime}}(l)+1 \leq i \leq\left[\frac{\Theta^{p}}{\vartheta^{p}}\right]\right)$ will be $\theta^{p *}+2 \theta^{p *} \cdot\left(\eta^{p, t^{\prime}}(l)-1\right)+2 \dot{\theta}^{p *}(l) \cdot\left(i-\eta^{p, t^{\prime}}(l)\right)$. Thus, the response cost of $\eta^{p, t^{\prime}}(l)+1^{\text {th }}$ to $\left\lceil\left.\frac{\Theta^{p}}{\vartheta^{p}}\right|^{\text {th }}\right.$ deliveries would be $\sum_{p \in P} \sum_{i=\eta^{p, t^{\prime}}(l)+1}^{\left\lceil\frac{\Theta^{p}}{\vartheta^{p}}\right\rceil} \vartheta^{p} . c^{p}\left(\theta^{p *}+\right.$ $\left.2 \theta^{p *} \cdot\left(\eta^{p, t^{\prime}}(l)-1\right)+2 \dot{\theta}^{p *}(l) \cdot\left(i-\eta^{p, t^{\prime}}(l)\right)\right)$. This means that recovering link $l$ will make $\sum_{p \in P} \sum_{i=\eta^{p, t^{\prime}}(l)+1}^{\left\lceil\frac{\Theta^{p}}{\vartheta^{p}}\right\rceil} \vartheta^{p} \cdot c^{p}\left(\theta^{p *}+2 \theta^{p *}(i-1)\right)-\sum_{p \in P} \sum_{i=\eta^{p, t^{\prime}}(l)+1}^{\left\lceil\Theta^{p}\right.} \vartheta^{p} \cdot c^{p}\left(\theta^{p *}+\right.$ $\left.2 \theta^{p *} \cdot\left(\eta^{p, t^{\prime}}(l)-1\right)+2 \dot{\theta}^{p *}(l) \cdot\left(i-\eta^{p, t^{\prime}}(l)\right)\right)$ reduction in the total response cost. 


\section{APPENDIX C}

In Model P1, all the roads of the network have similar priorities for damage assessment. Sometimes, some roads of the network are more prone to be damaged in disasters. Roads located beside oceans, seas, rivers, or epicenters or roads ending at the population centers located in highly disaster-prone regions have higher probabilities to be damaged by disasters. In this case, there are two groups of roads in the network: the set of prioritized roads $\left(L^{P} \subset L\right)$ and the set of nonprioritized roads $\left(\hat{L}^{U}=\hat{L}-\hat{L}^{P}\right)$. Binary parameter $\beta_{(n, m)}(\forall n, m \in \hat{N})$ is equal to 1 if the link $(n, m) \in \hat{L}^{P}$; and 0 otherwise $\left((n, m) \in \hat{L}^{U}\right)$. While $\Psi$ determines the scan completion time for the whole network, a new variable such as $\Omega$ is defined to determine the scan completion time for the prioritized roads:

$\Omega \geq \beta_{(n, m)} \cdot \sum_{t=1}^{|T|} t \cdot w_{(\overrightarrow{n, m})}^{t} \quad(\forall n, m \in \hat{N})$

To minimize the scan completion time for the prioritized roads, the objective function $(2 \mathrm{C})$ should be added to Model P1:

$$
\operatorname{MIN} \quad Z_{5}=\Omega
$$

Giving a higher weight to the third objective function compared to the second objective function makes Model P1 + objective function (2C) + constraint (1C) scan the prioritized roads prior to the non-prioritized roads. In the case with two scan completion times ( $\Psi$ and $\Omega$ ), the distance of the shortest path between a pair of adjacent charging stations would be less than or equal to $\operatorname{Min}\{2 \Omega, d\}$ if a prioritized road is located in the path and would be less than or equal to $\operatorname{Min}\{2 \Psi, d\}$ if a prioritized road does not exist in the path. This issue complicates locating charging stations in Proposition 2 and the UAV walks determination by the heuristic approach. To make the results usable for road networks with prioritized roads, we suggest a second reformation in the

unified network $G$. Based on this reformation, $\Psi-\Omega$ number of dummy nodes should be added to 
the unified links included in the prioritized roads. Assuming that roads $\left(n_{4}, n_{5}\right)$ and $\left(n_{1}, n_{5}\right)$ are the prioritized roads in the sample road network of Figure 5 and $\Psi-\Omega=1$, the structure of the network after the second reformation would be as Figure 1C.

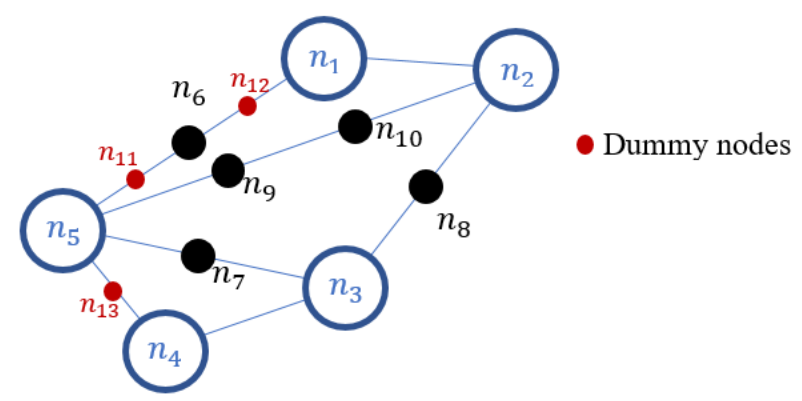

Figure 1C. The structure of the sample network after the second reformation.

Note that charging stations cannot be located at dummy nodes. These nodes are added to make sure that there are at most $\operatorname{Min}\{2 \Psi, d\}$ links between any pair of adjacent charging stations. Using this approach, we can neutralize the impact of prioritized roads and having two different completion times on Model P2 in Proposition 2. 